\title{
Diagnostic Computation of Moisture Budgets in the ERA-Interim Reanalysis with Reference to Analysis of CMIP-Archived Atmospheric Model Data*
}

\author{
Richard SEAGER AND NAOMI HeNDERSON \\ Lamont Doherty Earth Observatory, Columbia University, Palisades, New York
}

(Manuscript received 21 December 2012, in final form 11 April 2013)

\begin{abstract}
The diagnostic evaluation of moisture budgets in archived atmosphere model data is examined. Sources of error in diagnostic computation can arise from the use of numerical methods different from those used in the atmosphere model, the time and vertical resolution of the archived data, and data availability. These sources of error are assessed using the climatological moisture balance in the European Centre for Medium-Range Weather Forecasts Interim Re-Analysis (ERA-Interim) that archives vertically integrated moisture fluxes and convergence. The largest single source of error arises from the diagnostic evaluation of divergence. The chosen second-order accurate centered finite difference scheme applied to the actual vertically integrated moisture fluxes leads to significant differences from the ERA-Interim reported moisture convergence. Using daily data, instead of 6-hourly data, leads to an underestimation of the patterns of moisture divergence and convergence by midlatitude transient eddies. A larger and more widespread error occurs when the vertical resolution of the model data is reduced to the 8 levels that is quite common for daily data archived for the Coupled Model Intercomparison Project (CMIP). Dividing moisture divergence into components due to the divergent flow and advection requires bringing the divergence operator inside the vertical integral, which introduces a surface term for which a means of accurate evaluation is developed. The analysis of errors is extended to the case of the spring 1993 Mississippi valley floods, the causes of which are discussed. For future archiving of data (e.g., by CMIP), it is recommended that monthly means of time-step-resolution flowhumidity covariances be archived at high vertical resolution.
\end{abstract}

\section{Introduction}

Droughts and floods are some of the main disruptors of human life causing a never-ending sequence of death, destruction, suffering, hunger, disease, and economic devastation (see references in Cutter et al. 2009). As climate change driven by rising greenhouse gases proceeds, there will be additional hazards caused by both changes in the natural variability and changes in the mean precipitation distributions, as some tropical and mid- to highlatitude areas get wetter and subtropical dry areas get drier and expand (Allen and Ingram 2002; Held and Soden 2006; Solomon et al. 2007; Seager et al. 2010b, 2012).

\footnotetext{
* Lamont Doherty Earth Observatory Contribution Number 7714.
}

Corresponding author address: Richard Seager, Lamont Doherty Earth Observatory of Columbia University, 61 Route 9W, Palisades, NY 10964.

E-mail: seager@Ideo.columbia.edu
As for naturally occurring droughts and floods, changes in the mean precipitation distribution are caused by changes in the transport of water vapor in the atmosphere that create precipitation anomalies that either deprive areas of water or cause an excess. That is, the atmospheric branch of the hydrological cycle is the key phenomena where these risks to human livelihood originate.

Humans, being naturally curious, have long sought to determine the causes of droughts, pluvials, and floods relating them to the responsible changes in atmospheric circulation and water vapor transports. However, ultimately, we need to attempt to anticipate such events in advance so that preparations can be made and the worst impacts avoided. This is true both for the case of natural events occurring on the daily to decadal time scale and also for the more slowly evolving effect of hydroclimate change. In both cases, prediction or projection depends on the use of numerical climate models. Understanding then comes into play as a means of assessing how reliable predictions and projections are, given the fidelity with which the models simulate the important processes. 
For example, drought over southern North America during La Niña events fundamentally depends on moisture divergence anomalies caused by mean flow anomalies (Seager et al. 2005; Seager and Naik 2012), with the latter tightly coupled to changes in the North Pacific storm track (Seager et al. 2010a; Harnik et al. 2010).

Understanding of the causes of floods and droughts and of ongoing hydroclimate change requires a detailed analysis of the atmospheric moisture budget and the linking of this to changes in the atmospheric circulation and ultimately the atmospheric and planetary energy budget. This is not very easy to do either in atmospheric models or gridded, model-based reanalyses of atmospheric observations. In both cases, the models numerically integrate forward a moisture equation designed to best conserve moisture and to preserve a long-term mean balance between precipitation $P$, surface evaporation $E$, and the vertically integrated moisture convergence, although, in the case of reanalyses, the moisture field is also constrained, directly or indirectly, by observations. However, analyses of the causes of hydroclimate variability and change are done diagnostically, after the model has run, using saved data from the model. Typically this data includes velocities and specific humidity on a three-dimensional spatial grid as well as surface pressure, $P$, and $E$. The data may be saved at 6-hourly, daily, or monthly temporal resolutions but never at the time step of the model, and only rarely are monthly means of covariances between quantities (themselves evaluated variously using time step, four times daily, daily mean data, etc.) saved. Also the data are only sometimes saved on the native model grid and have often been interpolated to standard pressure levels with varying degrees of vertical resolution. Many efforts have been used to diagnose the moisture budget in reanalyses using pressure level data (e.g., Trenberth and Guillemot 1995; Trenberth 1997). Trenberth and Guillemot (1998) and Seneviratne et al. (2004) recommend performing moisture budget computations at the highest vertical resolution possible on the native model grid. While such data are becoming increasingly available, this is rarely universally practical with archives of data from multiple models such as those within phase 5 of the Coupled Model Intercomparison Project (CMIP5; Taylor et al. 2012).

The task of the researcher is, more commonly, to analyze the causes of hydroclimatic events using these incomplete model datasets. At the simplest level, the researcher will then discover that, in the long-term mean, the modelreported $P-E$ cannot be made to balance the convergence of the vertically integrated moisture flux, no matter how the latter is calculated. However, even if it did balance, this would not be very enlightening. The main goal of such work is to go further and determine what the causes of the moisture convergence or divergence anomalies are and, therefore, break it down into components due to changes in mean circulation, specific humidity, and transient eddies (e.g., Huang et al. 2005; Seager et al. 2010b; Seager and Naik 2012; Seager et al. 2012; Nakamura et al. 2013). To do this requires further analysis of the moisture budget and creates a new set of problems, as we shall see.

The point of this paper is to provide a detailed and thorough assessment of the errors introduced in diagnostic analyses of the moisture budget and how these depend on the temporal and spatial resolution of the data and what additional errors are introduced in attempts to break down moisture convergence into constituent parts. We also aim to provide guidance as to the best possible way to numerically evaluate the moisture budget with existing model data and suggest improvements for the archiving of model and reanalysis data in the future that will allow improved accuracy in diagnostic computations. To this effect, we will consider the climatological moisture budget and then apply the lessons learned to the moisture budget during a major hydroclimatic anomalythat of the Mississippi floods of late spring-early summer 1993 - and show that, budget errors notwithstanding, it is possible to use the chosen reanalysis to elucidate the physical mechanisms that led to the flood.

\section{Reanalyses data used}

For demonstration purposes we use the European Centre for Medium-Range Weather Forecasts (ECMWF) Interim Re-Analysis (ERA-Interim) (Berrisford et al. 2011b,a; Dee et al. 2011), which is the latest of the ECMWF reanalyses. ERA-Interim covers the post-1979 period. It assimilates cloud and rain-affected satellite irradiances and has a greatly improved representation of the hydrological cycle relative to its precursor, the 40-yr ECMWF Re-Analysis (ERA-40). This makes it good for our purpose. Berrisford et al. (2011a) discuss the conservation of moisture in the ERA-Interim and conclude that mass adjustment of the moisture divergence is not necessary, and this was not done to the reported fields. Also, ERA-Interim provides the divergence of the vertically integrated moisture transport as data output: that is, this provides the actual value of the quantity we are trying to evaluate diagnostically from archived model or reanalysis data. However, it should be noted, in part because of the assimilation scheme, this quantity does not balance the ERA-Interim $P-E$, even after accounting for the change over time of the vertically integrated specific humidity (see Trenberth et al. 2011). ERA-Interim is based on an atmospheric model and reanalysis system with 60 levels in the vertical with a top level at $0.1 \mathrm{mb}$; a T255 spherical harmonic representation; and for surface and gridpoint fields, a reduced 
Gaussian grid with an about 79-km spacing (Berrisford et al. 2011b). However, the highest-resolution calculations reported here are performed on data that was archived by ECMWF on a regular $0.75^{\circ}$ grid with 37 model levels. At the time of writing, not all the 6-hourly pressure level data needed for our calculations were available on the $0.75^{\circ}$ grid. Further, it would have been impractical to download and store all the data we needed at this temporal and full spatial resolution, and therefore, for most of the calculations, we use the $1.5^{\circ}$ longitude by latitude data also archived by ECMWF.

\section{Diagnostic computation of the moisture budget in atmosphere models}

Most models use a terrain-following vertical coordinate. The $\sigma$ coordinate, with $p=\sigma p_{s}$, where $p$ is pressure and $p_{s}$ is its surface value, was the first such coordinate, but more commonly used today is a hybrid vertical coordinate $\xi$, which preserves $\xi=0$ at $p=0$ and $\xi=1$ at $p=p_{s}$ but with the pressure at model level $k$ given by $p_{k}=A_{k}+B_{k} p_{s}$, where $A_{k}$ and $B_{k}$ are constants. The hybrid vertical coordinate is usually set up to vary from a terrain-following coordinate in the lower troposphere to a $p$ coordinate in the stratosphere. On the other hand, model data are commonly archived on standard pressure levels necessitating the use of a $p$ coordinate in diagnostic analysis. To deal with both these vertical coordinate systems, we begin with a generalized vertical coordinate $\eta$ (see Konor and Arakawa 1997), for which the material derivative of a quantity is given by

$$
\frac{D}{D t}=\left(\frac{\partial}{\partial t}\right)_{\eta}+\mathbf{u} \cdot \nabla_{\eta}+\dot{\eta} \frac{\partial}{\partial \eta}
$$

where $\dot{\eta}=D \eta / D t$.

In this vertical coordinate, the moisture equation is (dropping $\eta$ subscripts)

$$
\frac{\partial q}{\partial t}+\nabla \cdot(\mathbf{u} q)+\dot{\eta} \frac{\partial q}{\partial \eta}=e-c
$$

where $q$ is specific humidity, $\mathbf{u}$ is the velocity vector along $\eta$ surfaces, and $e$ and $c$ are evaporation and condensation. We use spherical coordinates so the divergence of moisture is given by

$$
\boldsymbol{\nabla} \cdot(\mathbf{u} q)=\frac{1}{a \cos \phi}\left[\frac{\partial(u q)}{\partial \lambda}+\frac{\partial(v q \cos \phi)}{\partial \phi}\right],
$$

where $u$ and $v$ are the zonal and meridional components of velocity, $a$ is the radius of the earth, $\lambda$ is longitude, and $\phi$ is latitude. The continuity equation is

$$
\frac{\partial}{\partial t} \frac{\partial p}{\partial \eta}+\nabla \cdot\left(\mathbf{u} \frac{\partial p}{\partial \eta}\right)+\frac{\partial}{\partial \eta}\left(\dot{\eta} \frac{\partial p}{\partial \eta}\right)=0
$$

These can be combined into the flux form of the humidity equation,

$\frac{\partial}{\partial t}\left(q \frac{\partial p}{\partial \eta}\right)+\nabla \cdot\left(\mathbf{u} q \frac{\partial p}{\partial \eta}\right)+\frac{\partial}{\partial \eta}\left(q \dot{\eta} \frac{\partial p}{\partial \eta}\right)=\frac{\partial p}{\partial \eta}(e-c)$.

This equation can be vertically integrated to derive a relation for the precipitation minus surface evaporation $P-E$,

$$
\begin{aligned}
P-E= & -\frac{1}{g \rho_{w}} \int_{0}^{1} \frac{\partial}{\partial t}\left(q \frac{\partial p}{\partial \eta}\right) d \eta \\
& -\frac{1}{g \rho_{w}} \int_{0}^{1} \nabla \cdot\left(\mathbf{u} q \frac{\partial p}{\partial \eta}\right) d \eta,
\end{aligned}
$$

where $g$ is the acceleration due to gravity and $\rho_{w}$ is the density of water: the inclusion of which mean that $P-E$ is in units of meters per second (or mm day ${ }^{-1}$, as will be shown in the figures). Since the limits of integration on $\eta$ are independent of space and time, this can be rewritten with the time derivative and divergence operator outside of the integral as

$$
P-E=-\frac{1}{g \rho_{w}} \frac{\partial}{\partial t} \int_{0}^{1}\left(q \frac{\partial p}{\partial \eta}\right) d \eta-\frac{1}{g \rho_{w}} \nabla \cdot \int_{0}^{1}\left(\mathbf{u} q \frac{\partial p}{\partial \eta}\right) d \eta .
$$

In the case of data provided on pressure levels, we revert to a $p$ coordinate for which Eq. (5) becomes

$$
\frac{\partial q}{\partial t}+\nabla \cdot(\mathbf{u} q)+\frac{\partial}{\partial p}(\omega q)=e-c .
$$

The $p$-coordinate flux form moisture equation can be vertically integrated from the surface pressure $p_{s}$ to the top of the atmosphere to derive

$$
\begin{aligned}
P-E= & -\frac{1}{g \rho_{w}} \int_{0}^{p} \frac{\partial q}{\partial t} d p-\frac{1}{g \rho_{w}} \int_{0}^{p_{s}} \nabla \cdot(\mathbf{u} q) d p \\
& -\frac{1}{g \rho_{w}} \omega_{s} q_{s},
\end{aligned}
$$

where the subscript $s$ refers to surface quantities. Noting that

$$
\begin{gathered}
\omega_{s}=\frac{\partial p_{s}}{\partial t}+\mathbf{u}_{s} \cdot \nabla p_{s} \\
\int_{0}^{p_{s}} \frac{\partial q}{\partial t} d p=\frac{\partial}{\partial t} \int_{0}^{p_{s}} q d p-q_{s} \frac{\partial p_{s}}{\partial t}, \quad \text { and }
\end{gathered}
$$




$$
\int_{0}^{p_{s}} \nabla \cdot(\mathbf{u} q) d p=\nabla \cdot \int_{0}^{p_{s}} \mathbf{u} q d p-q_{s} \mathbf{u}_{s} \cdot \nabla p_{s},
$$

we derive

$$
P-E=-\frac{1}{g \rho_{w}} \frac{\partial}{\partial t} \int_{0}^{p_{s}} q d p-\frac{1}{g \rho_{w}} \nabla \cdot \int_{0}^{p_{s}} \mathbf{u} q d p .
$$

This is the form of the moisture budget equation that we focus most of the analysis on. However, this form only allows understanding of the moisture budget (and its variations) to advance so far. Note that the divergence operates on the vertically integrated moisture field and does not allow a breakdown of the moisture convergence into a part due to the mass convergence and a part due to advection of humidity gradients. Therefore, an alternative form is often presented,

$$
\begin{aligned}
P-E= & -\frac{1}{g \rho_{w}} \frac{\partial}{\partial t} \int_{0}^{p_{s}} q d p-\frac{1}{g \rho_{w}} \int_{0}^{p_{s}} \nabla \cdot(\mathbf{u} q) d p \\
& -\frac{1}{g \rho_{w}} q_{s} \mathbf{u}_{s} \cdot \nabla p_{s},
\end{aligned}
$$

which allows the divergence to be broken down into parts related to a divergent flow $q \nabla \cdot \mathbf{u}$ and a part related to advection $\mathbf{u} \cdot \nabla q$ : namely,

$$
\begin{aligned}
P-E= & -\frac{1}{g \rho_{w}} \frac{\partial}{\partial t} \int_{0}^{p_{s}} q d p-\frac{1}{g \rho_{w}} \int_{0}^{p_{s}}(q \nabla \cdot \mathbf{u}+\mathbf{u} \cdot \nabla q) d p \\
& -\frac{1}{g \rho_{w}} q_{s} \mathbf{u}_{s} \cdot \nabla p_{s} .
\end{aligned}
$$

Here, the separation into components of moisture divergence due to divergent flow and advection is only allowed by bringing the divergence operator inside the vertical integral and hence introduces a boundary term $q_{s} \mathbf{u}_{s} \cdot \nabla p_{s}$ that also needs to be accounted for [which is sometimes discussed (Seager and Vecchi 2010; Seager et al. 2010b) but is also often ignored (Seager et al. 2007)].

These equations have been written in continuous form but in models will be evaluated using various numerical methods. For example, the model that ERA-Interim is based upon uses a finite difference method to evaluate vertical derivatives and a semiLagrangian method to determine advective tendencies (ECMWF 2012). Other models use three-dimensional semi-Lagrangian methods. The humidity tendencies induced by these schemes cannot be reproduced using archived data that already include the effect of the advection even if the data were archived at the model time step. A numerical method needs to be chosen to evaluate the terms in the moisture equation with the additional goal that it is general enough to be applicable to a variety of reanalyses and/or models.

The vertically integrated moisture transport is approximated by

$$
\int_{0}^{p_{s}}(\mathbf{u} q) d p \approx \sum_{k=1}^{K_{i, j}} \mathbf{u}_{k} q_{k} \Delta p_{k},
$$

where the summation is over vertical layers $k$, of which there are $K_{i, j}$, with $i$ and $j$ indicating the longitude and latitude location of grid points. In the original $\eta$ coordinates $K_{i, j}$ is the same at all grid points but for archived pressure level data $K_{i, j}$ will depend on longitude and latitude. The divergence operator on a two-dimensional vector $\mathbf{F}$ is evaluated via

$$
\begin{aligned}
\nabla_{f} \cdot \mathbf{F} \approx & \frac{1}{a \cos \phi_{j}}\left\{\frac{1}{\lambda_{i+1, j}-\lambda_{i-1, j}}\left[\left(\lambda_{i, j}-\lambda_{i-1, j}\right) \frac{F_{i+1, j}^{\lambda}-F_{i, j}^{\lambda}}{\lambda_{i+1, j}-\lambda_{i, j}}+\left(\lambda_{i+1, j}-\lambda_{i, j}\right) \frac{F_{i, j}^{\lambda}-F_{i-1, j}^{\lambda}}{\lambda_{i, j}-\lambda_{i-1, j}}\right]\right. \\
& \left.+\frac{1}{\phi_{i, j+1}-\phi_{i, j-1}}\left[\left(\phi_{i, j}-\phi_{i, j-1}\right) \frac{\cos \phi_{j+1} F_{i, j+1}^{\phi}-\cos \phi_{j} F_{i, j}^{\phi}}{\phi_{i, j+1}-\phi_{i, j}}+\left(\phi_{i, j+1}-\phi_{i, j}\right) \frac{\cos \phi_{j} F_{i, j}^{\phi}-\cos \phi_{j-1} F_{i, j-1}^{\phi}}{\phi_{i, j}-\phi_{i, j-1}}\right]\right\},
\end{aligned}
$$

where $F^{\lambda}$ and $F^{\phi}$ indicate the components of $\mathbf{F}$ in the longitude and latitude directions and $\nabla_{f}$ is used to indicate a finite difference approximation to the divergence operator on a longitude-latitude grid. To evaluate moisture divergence, $\mathbf{F}_{i, j}$ is given by

$$
\mathbf{F}_{i, j}=\sum_{k=1}^{K_{i, j}} \mathbf{u}_{i, j, k} q_{i, j, k} \Delta p_{i, j, k} .
$$

To evaluate the divergence at grid point $(i, j)$, Eq. (17) computes centered differences at midpoints to the east and west and to the north and south and then linearly interpolates these in the $\lambda$ and $\phi$ directions back to the $(i, j)$ point. This therefore allows for the case of uneven grid spacing (quite common in CMIP models in the $\phi$ direction). In the case of an even grid, which the ERAInterim data are served on, Eq. (17) reduces to the familiar form 


$$
\begin{aligned}
\nabla_{f} \cdot \mathbf{F} \approx & \frac{1}{a \cos \phi_{j}}\left(\frac{F_{i+1, j}^{\lambda}-F_{i-1, j}^{\lambda}}{\lambda_{i+1, j}-\lambda_{i-1, j}}\right. \\
& \left.+\frac{\cos \phi_{j+1} F_{i, j+1}^{\phi}-\cos \phi_{j-1} F_{i, j-1}^{\phi}}{\phi_{i, j+1}-\phi_{i, j-1}}\right) .
\end{aligned}
$$

The vertical integration goes down to the surface pressure as follows: The pressure thickness of the lowest layer is equal to the surface pressure minus the pressure at the first reported pressure level above and, within this layer, the values of $\mathbf{u}$ and $q$ used are the ones of the first pressure level above the surface pressure value. All of these integration and differentiation approximations introduce errors. In addition, the time resolution of the diagnostic computation will also causes errors if it does not conform to the actual time step of the model. For example, a calculation done with 6-hourly data would be expected to be more accurate than one done with daily data.

\section{Evaluation of sources of error in diagnostic moisture budget calculations}

Here we assess the relative importance of the approximations introduced into diagnostic computation of moisture budgets as detailed in the prior section.

\section{a. Patterns of $P-E$ and divergence of ERA-Interim- reported vertically integrated moisture divergence}

First of all, the ERA-Interim reports within its data archive what is called the vertically integrated moisture divergence, which we multiply by -1 to convert to moisture convergence (MC). ERA-Interim also reports the vertically integrated moisture flux (VIMF). These correspond to

$$
\begin{aligned}
\mathrm{MC} & =-\frac{1}{g \rho_{w}} \sum_{k=1}^{K} \nabla \cdot\left(\mathbf{u} q \frac{\partial p}{\partial \eta} \Delta \eta\right)_{k} \\
& =-\frac{1}{g \rho_{w}} \nabla \cdot \sum_{k=1}^{K}\left(\mathbf{u} q \frac{\partial p}{\partial \eta} \Delta \eta\right)_{k} \\
& =-\frac{1}{g \rho_{w}} \nabla \cdot \mathrm{VIMF},
\end{aligned}
$$

with the vertical sum done on the model $\eta$ grid, as indicated by use of $(\partial p / \partial \eta) \Delta \eta$, over the $K$ model layers. Note that since this is evaluated on the model $\eta$ grid it does not matter whether the divergence operator is inside or outside the vertical sum. ECMWF report MC and VIMF as both monthly means of daily means and also as 6-hourly values with the daily mean equal to the average of the four 6-hourly values within that day.
Using a double overbar to indicate climatological monthly means, Fig. 1 shows the climatological monthly means for January and July of $\overline{\overline{\mathrm{MC}}}$ and precipitation minus evaporation $\overline{\overline{P-E}}$ for the ERA-Interim dataset, as well as their difference. Not surprisingly, there is a rather close balance between these two but the difference shows that this is not a perfect match by any means. In reality, vertically integrated moisture divergence on the model grid should differ from $P-E$ by the change in vertically integrated moisture [Eq. (7)]. Hence, we also show this in Fig. 1, where it is evaluated for each month as the ERA-Interim-reported vertically integrated moisture content for the first day of the next month minus that for the first of the month itself. The change in moisture storage shows the expected seasonal cycle (moistening in the summer hemisphere and drying in the winter hemisphere), but this pattern is quite different from the $\overline{\overline{P-E}}-\overline{\overline{\mathrm{MC}}}$ one. The imbalance is very similar in pattern to that shown by Berrisford et al. (2011a).

Consequently, even though the reanalysis reports a vertically integrated moisture divergence, this does not balance the sum of model $\overline{\overline{P-E}}$ and change in moisture storage. There are three possible reasons for this. One is that Eq. (20) is an approximation to the moisture convergence the model effectively sees. This is because the ECMWF model actually updates its humidity field by applying a semi-Lagrangian scheme to an advective form of the moisture equation. As such, moisture divergence does not need to be evaluated in the updating of the model. In contrast, to derive MC as a diagnostic, the moisture divergence is evaluated in spectral space the same way that mass divergence is computed in the model to evaluate vertical velocities (Berrisford et al. 2011a). Another reason for an imbalance is that the ECMWF model contains a moisture diffusion along $\eta$ surfaces (ECMWF 2012) but the $q$ tendencies induced by this are not saved or known (and also cannot be computed from the humidity field after the fact). The third reason is that the reported $P, E, q$, and MC fields have been influenced by the data assimilation scheme such that the moisture budget [Eq. (6)] need no longer be in balance because of so-called analysis increments (Trenberth et al. 2011).

\section{b. Error introduced in evaluation of time mean divergence of vertically integrated moisture flux}

The imbalance between $P-E$, moisture storage, and $\mathrm{MC}$ in ERA-Interim is not of immediate concern to us. In climate models, these will balance more closely because the moisture budget is closed because of the absence of analysis increments. Hence, our main effort is to assess how well the divergence of vertically integrated 
Moisture Convergence, $\overline{\overline{M C}}$
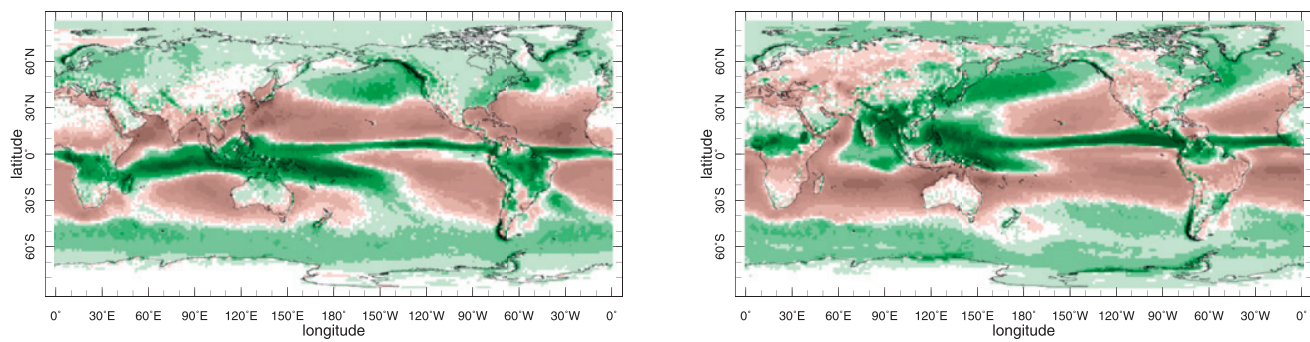

$\overline{\overline{P-E}}$
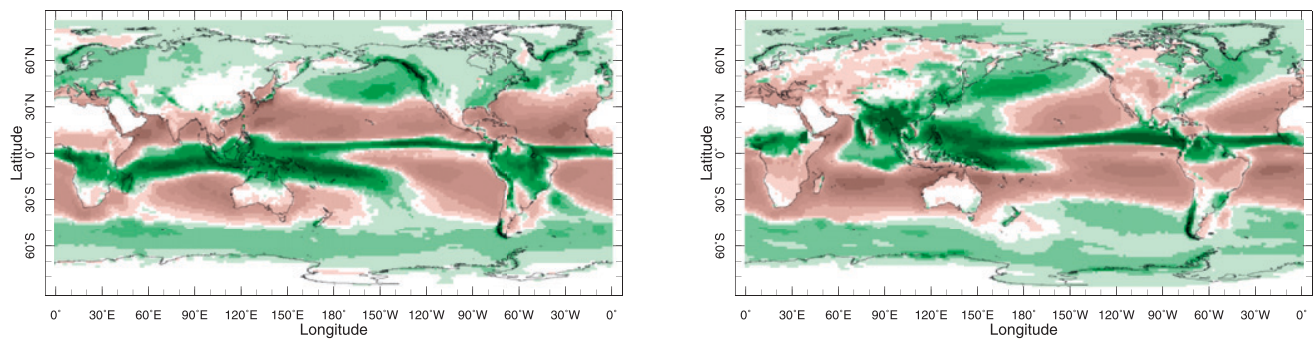

$\overline{\overline{P-E}}-\overline{\overline{M C}}$
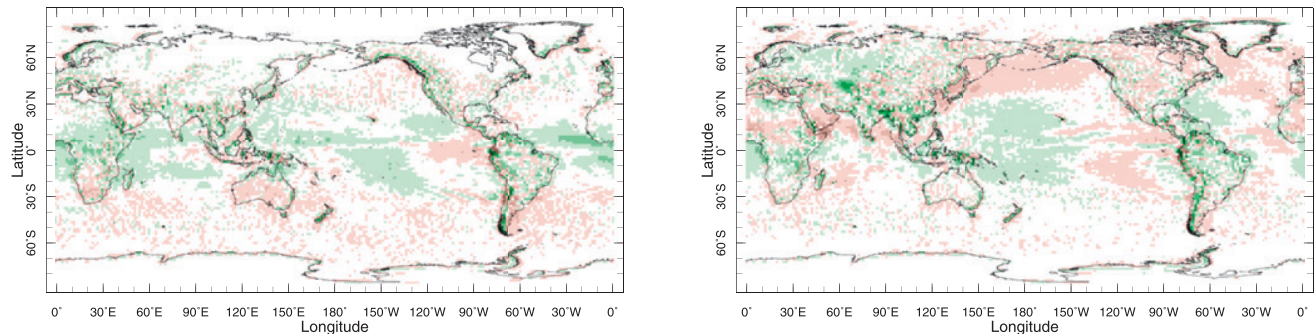

Change in Vertically Integrated Moisture Content
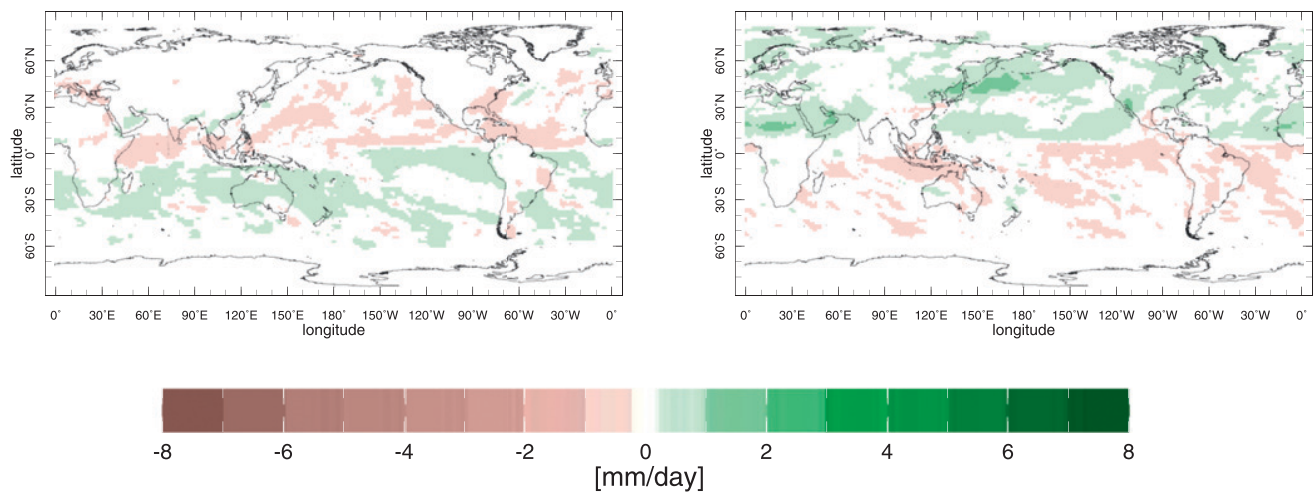

FIG. 1. The (left) January and (right) July climatologies of the ERA-Interim-reported (top) vertically integrated moisture divergence $\overline{\overline{\mathrm{MC}}}$, (top middle) $\overline{\overline{P-E}}$, (bottom middle) their difference, and (bottom) the change in moisture storage computed from the reported vertically integrated moisture content. (Units are mm day ${ }^{-1}$.) 
moisture can be evaluated diagnostically using archived data. That is, how well can the ERA-Interim-reported MC itself be approximated from archived $\mathbf{u}$ and $q$ on pressure levels together with $p_{s}$ ? As discussed, errors will be introduced in the evaluation of the divergence, in the evaluation of the vertical integral and by the time resolution of the data, which will each be treated in turn.

\section{1) ERROR FROM EVALUATION OF DIVERGENCE}

ERA-Interim reports the VIMF and its convergence, MC. Hence, by applying to VIMF the simple centered difference divergence operator as in Eq. (17), we determine the error introduced relative to the ERAInterim-reported value. That is, we evaluate

$$
-\frac{1}{g \rho_{w}} \nabla \cdot \overline{\overline{\mathrm{VIMF}}} \quad \text { cf. } \quad-\frac{1}{g \rho_{w}} \nabla_{f} \cdot \overline{\overline{\mathrm{VIMF}}}
$$

Figure 2 shows this difference. Most of the analyses to follow are on the $1.5^{\circ}$ grid and these results are shown in the middle row of Fig. 2. The difference between the $1.5^{\circ}$ actual and diagnosed convergence is considerably larger than any subsequent errors introduced through decreases in temporal or vertical resolution. Errors introduced by the divergence operator approximation are concentrated in regions where the spatial gradients in the moisture convergence field are large. This is expected, as the errors in the $\nabla_{f}$ approximation will appear like derivatives of the divergence field. For example, the Pacific and Atlantic intertropical convergence zones (ITCZs), where the moisture convergence varies in strength and sign over small meridional distances, are regions of notable error. Coastal regions, where the moisture convergence also has strong gradients, and mountainous regions are other areas where the divergence approximation introduces notable errors.

The top row of Fig. 2 shows the same difference between reported and diagnosed moisture convergence when the $0.75^{\circ}$ grid data are used. This is much smaller than the error using the coarse-resolution data and makes clear that discretization error is a major source of error in the latter. However, even at the higher resolution, sizable errors in the diagnostic calculation occur, especially over land and regions of severe topography. To assess how coherent the errors are, in the bottom row of Fig. 2 we show a version of the error with the $1.5^{\circ}$ grid after one pass of a 1-2-1 spatial smoother. This effectively removes a lot of the error, as expected if it arises from discretization error, but notably leaves errors near key climatic features like the ITCZ.

Table 1 shows the climatological area averages of root-mean-square differences between monthly means of $-\left(1 / g \rho_{w}\right) \nabla_{f} \cdot \overline{\overline{\mathrm{VIMF}}}$ and both $\overline{\overline{\mathrm{MC}}}$ and the convergence of vertically integrated moisture as computed by us. These are all for the $1.5^{\circ}$ grid. It can be seen there that the largest error comes from the comparison of $\overline{\overline{\mathrm{MC}}}$ with $-\left(1 / g \rho_{w}\right) \nabla_{f} \cdot \overline{\overline{V I M F}}$ : that is, purely from the evaluation of divergence. The other root-mean-square errors in Table 1 are between quantities in which for both the divergence is computed by us as in Eq. (17) (see below) and therefore include only errors due to time or vertical resolution of that data. These are smaller than the error introduced by the divergence evaluation. This error can be made smaller by applying the finite difference divergence operator to data closer to the actual model resolution but not entirely removed. It should be recalled that moisture convergence is never actually computed during integration of the model, so it is not clear what the actual truth is and some level of disagreement has to accepted. The issue then becomes the extent to which it impacts any analysis of interest, a matter we address later.

\section{2) ERror INTRODUCED FROM USING TIME RESOLUTION OF ARCHIVED DATA}

We begin by considering how the moisture balance is impacted by the fact that the archived data are not at the model time step but are instead stored at the 6-hourly or perhaps daily time scale. In the case of ERA-Interim, the data are 6 hourly and hence ignore the covariance of $\mathbf{u}$ and $q$ at shorter time scales. To do this we show in Fig. 3 the quantity

$$
-\frac{1}{g \rho_{w}} \nabla_{f} \cdot \sum_{k=1}^{K} \overline{\overline{\mathbf{u}_{6, k} q_{6, k} \Delta p_{6, k}}}-\overline{\overline{\mathrm{MC}}},
$$

where the $i$ and $j$ subscripts have been dropped for simplicity and the subscript 6 indicates that this is evaluated using 6-hourly data for $\mathbf{u}, q$, and $p$. In this case errors are introduced both by the reduced time resolution of the data and by the vertical integration being performed by us (on 26 levels) rather than by ECMWF in a way presumably consistent with the model numerics. Quantitatively, the root-mean-square differences between the various diagnostic estimates of climatological MC and the actual ERA-Interim-reported values are given in Table 2. There it can be seen, by comparison to Table 1 , that $\overline{\overline{\mathrm{MC}}}$ is actually closer to the divergence of our vertically integrated moisture flux than it is to the divergence of the ERA-Interim-reported vertically integrated moisture flux. This is something we cannot explain, though it implies compensating errors in our computation of divergence and vertical integrals. Despite this nagging issue, Fig. 3 shows that, apart from a hint of systematic error near the ITCZ, the errors from time resolution and vertical integration appear randomly scattered around 


\section{January climatology}

\section{July climatology}

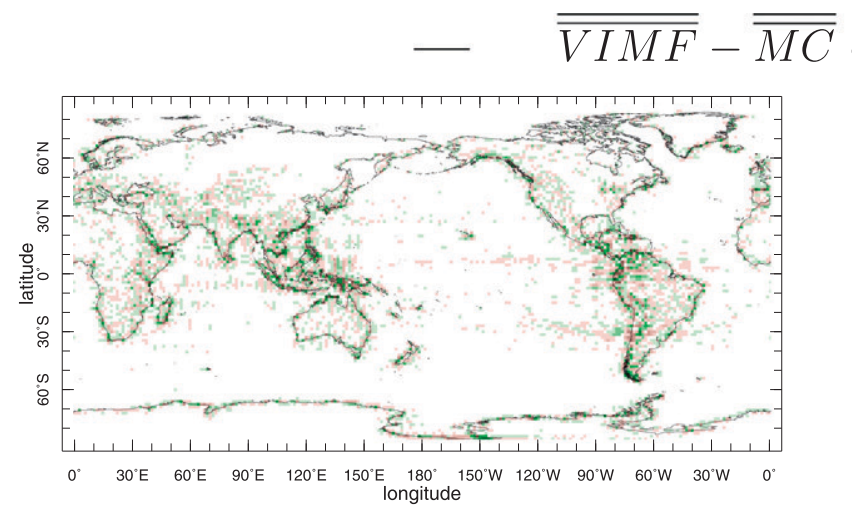

on $0.75^{\circ} \times 0.75^{\circ}$ grid
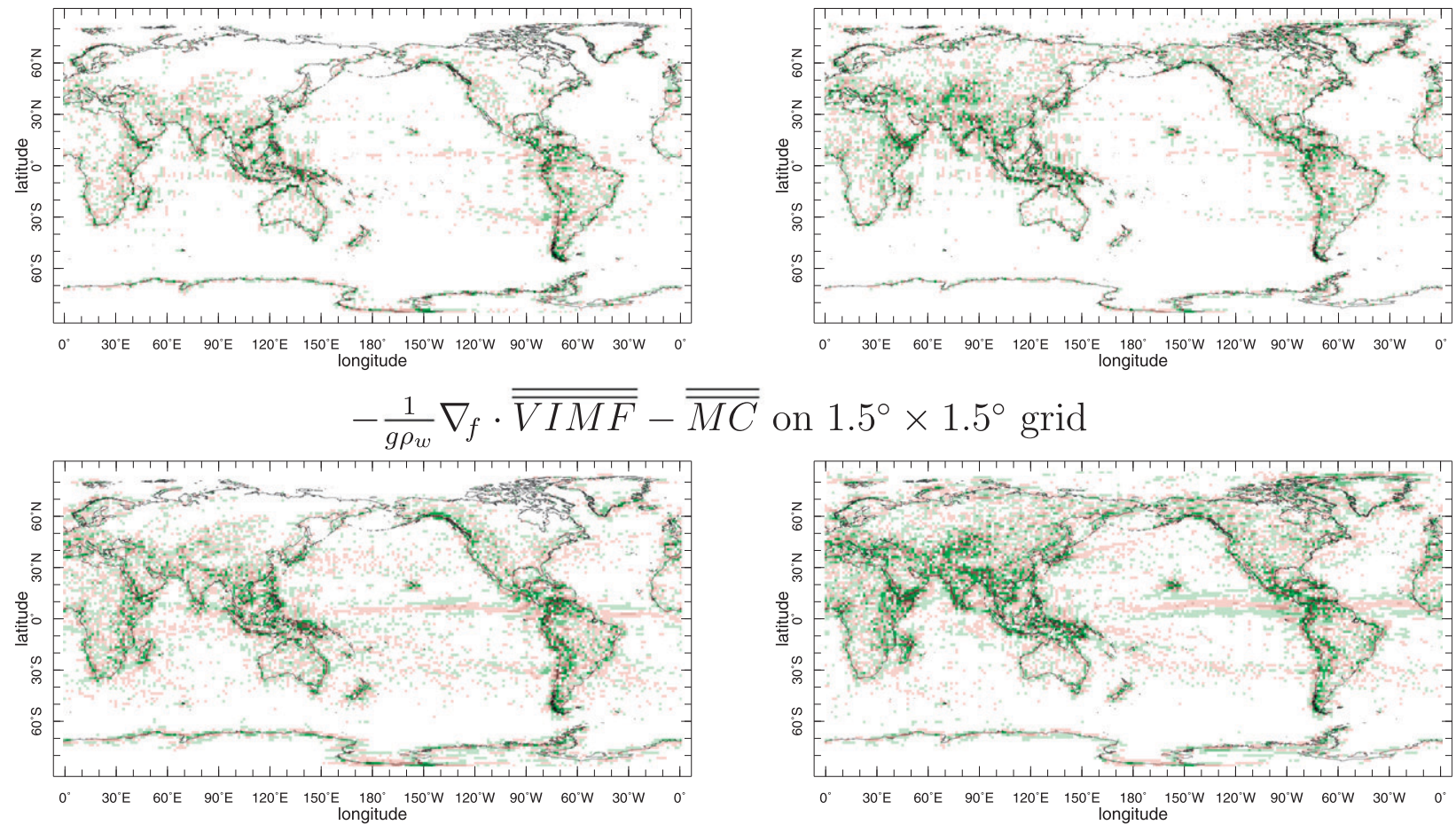

$$
-\frac{1}{g \rho_{w}} \nabla_{f} \cdot \overline{\overline{V I M F}}-\overline{\overline{M C}} \text { on } 1.5^{\circ} \times 1.5^{\circ} \text { grid, smoothed }
$$
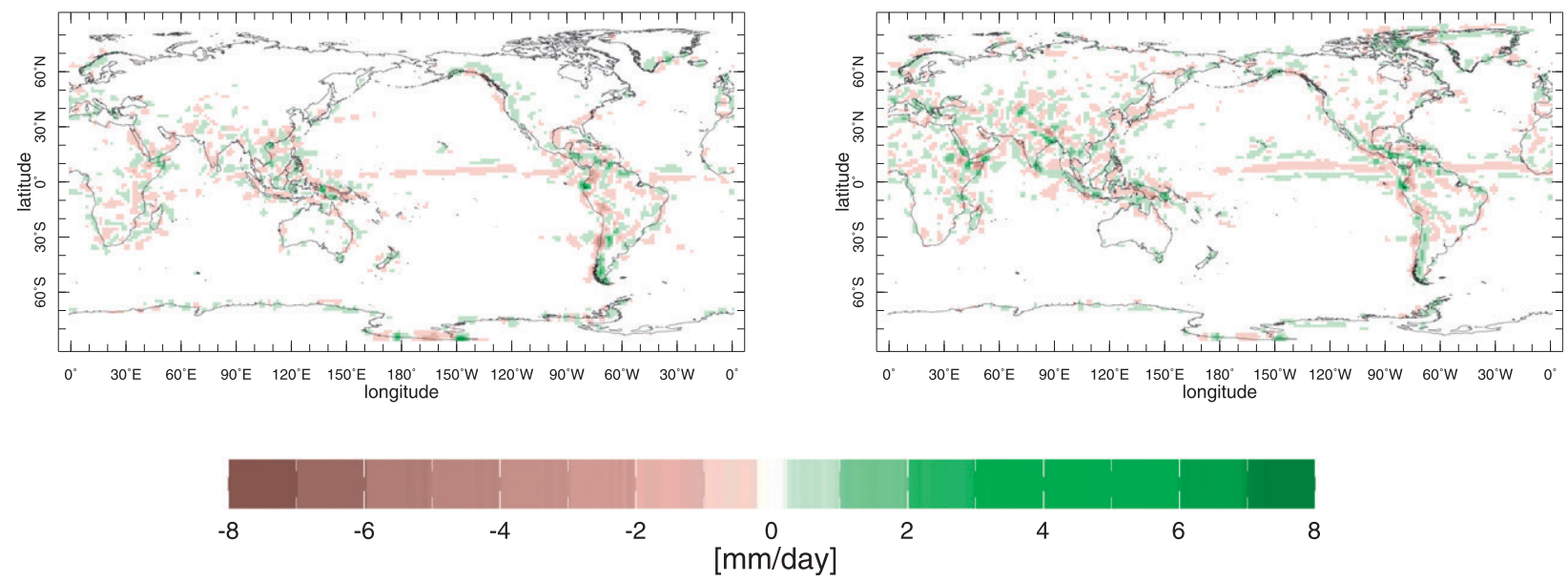

FIG. 2. (top) The (left) January and (right) July climatologies of the difference between the divergence of the ERA-Interim-reported vertically integrated moisture flux $\overline{\overline{\mathrm{VIMF}}}$ as evaluated using a centered finite difference scheme and the ERA-Interim-reported value $\overline{\overline{\nabla_{f} \cdot \text { VIMF }}}-\overline{\overline{\mathrm{MC}}}$ all on $0.75^{\circ}$ grid. (middle) As in (top), but on a $1.5^{\circ}$ grid. (bottom) As in (middle), but after application of one pass of a 1-2-1 spatial smoother. (Units are mm day ${ }^{-1}$.)

the globe. The ITCZ errors may be due to the existence in that region of transient storm systems with covarying winds and humidity on the less than 6-hourly time scale.
Figure 3 also shows the quantity

$$
-\frac{1}{g \rho_{w}} \nabla_{f} \cdot \sum_{k=1}^{K} \overline{\overline{\mathbf{u}_{d, k} q_{d, k} \Delta p_{d, k}}}-\overline{\overline{\mathrm{MC}}}
$$


TABLE 1. The long-term average of root-mean-square differences $\left(\mathrm{mm} \mathrm{day}^{-1}\right)$ between the monthly mean diagnostically computed convergence of ERA-Interim-reported vertically integrated moisture flux $\left(\nabla_{f}\right.$. VIMF $)$ and, in the left column, the ERA-Interim-reported monthly mean vertically integrated MC and, in the other columns to the right, diagnostically computed convergences of diagnostically computed monthly mean vertical moisture fluxes.

\begin{tabular}{lcccc}
\hline \hline \multicolumn{5}{c}{$\operatorname{RMS}\left[(\cdot)-\nabla_{f} \cdot\right.$ VIMF $]$} \\
\hline MC & $\begin{array}{c}\text { 6 hourly, } \\
\text { 26 levels }\end{array}$ & $\begin{array}{c}\text { 6 hourly, } \\
\text { 18 levels }\end{array}$ & $\begin{array}{c}\text { 6 hourly, } \\
\text { 8 levels }\end{array}$ \\
\hline Global & 1.31 & 0.93 & 1.04 & 1.89 \\
Land & 1.94 & 1.34 & 1.53 & 2.82 \\
Ocean & 0.95 & 0.70 & 0.76 & 1.35 \\
$30^{\circ} \mathrm{S}-30^{\circ} \mathrm{N}$ & 1.21 & 0.86 & 0.94 & 1.71 \\
$30^{\circ}-90^{\circ} \mathrm{N} / \mathrm{S}$ & 0.53 & 0.45 & 0.47 & 0.77 \\
\hline
\end{tabular}

where the $d$ subscript indicates this was evaluated with daily data. In this case, errors are systematic with too little moisture divergence at the subtropical edge of the midlatitudes and too little moisture convergence in the middle to high latitudes. This clearly represents an underestimation of poleward moisture transport by midlatitude transient eddies with the error arising from not sampling the subdaily covariance between the flow and the humidity. Since these midlatitude storms have characteristic time scales of a few to several days, it is reasonable that daily resolution data will be inadequate to capture their effects. This point is made clear in Fig. 3, where we show the difference between the 6-hourly and daily moisture convergence, with the former having stronger subtropical to midlatitude moisture transport with divergence on the subtropical side and convergence on the poleward side.

\section{3) ERROR FROM VERTICAL INTEGRATION USING FEWER PRESSURE LEVELS}

The calculations so far in which we performed the vertical integration used 26 vertical levels, which is more than is often available in archives of model data. Hence, we redo the integrations with daily data but with a degraded 18-level dataset that has fewer model levels near the surface. Figure 4 shows the difference between an 18-layer vertical integration of the moisture convergence and $\overline{\overline{\mathrm{MC}}}$ (which can be compared with Fig. 3 for the 26-layer case) and the difference between the 26- and 18-layer integrations, all using daily data. As expected, the errors are in general larger when using fewer layers, but these are restricted to land while differences over the ocean are small (also see Table 2). The increased error over land is because of less resolution in the lower atmosphere, where the moisture is located and also where vertical gradients of moisture are often large.

The 6-hourly data are really required for evaluating the transient contributions to moisture budgets, but archiving 6-hourly or even daily data for complete model runs at model vertical resolution places a considerable stress on data storage requirements and, once archived, on networks used to transfer data from the modeling groups that produce it to researchers elsewhere that analyze it. In many cases, therefore, the 6-hourly or daily data are archived on a subset of vertical levels to reduce the amount of data archived. For example, examining the current CMIP5 archive of 6-hourly and daily data, it was found that the 6-hourly data were typically only available on 3 vertical levels, obviously inadequate for moisture budget evaluation, and that daily data were available typically on 8 vertical levels. Hence, we next determined how closely an evaluation with daily data on 8 levels can match the actual convergence of vertically integrated moisture: that is, the comparison

$$
\overline{\overline{\mathrm{MC}}} \quad \text { cf. } \quad-\frac{1}{g \boldsymbol{\rho}_{w}} \nabla_{f} \cdot \sum_{k=1}^{8} \overline{\overline{\mathbf{u}_{d, k} q_{d, k} \Delta p_{d, k}}} .
$$

This comparison already includes the error in going to daily or 6-hourly data and the error in going from 26 to 18 levels and then introduces an additional error in going to 8 levels from 18 . However, we choose to show the total error in Fig. 4. Comparing to the 18-level data, the 8 -level case introduces significantly more error across the globe with notable errors appearing in the ITCZ regions and already existing errors over land becoming much larger. The degradation of the balance in the moisture budget when reducing the vertical resolution to only 8 levels is really quite striking.

\section{4) ERROR INTRODUCED BY IGNORING THE SUBMONTHLY VARIATIONS OF SURFACE PRESSURE}

Up to now the vertical integrals have been performed at the temporal resolution of the data (e.g., every $6 \mathrm{~h}$ or day) using the surface pressure at the same temporal resolution as the lower limit of integration. This allows for any covariation between flow fields, specific humidity, and surface pressure. However, it is our experience that high-temporal-resolution surface pressure data are not always available, so next we address the error introduced by first computing the time mean of the covariance of $\mathbf{u}$ and $q$ and then vertically integrating this using the time mean surface pressure. Introducing a single overbar to denote a monthly mean, we perform the comparisons 


\section{January climatology July climatology}
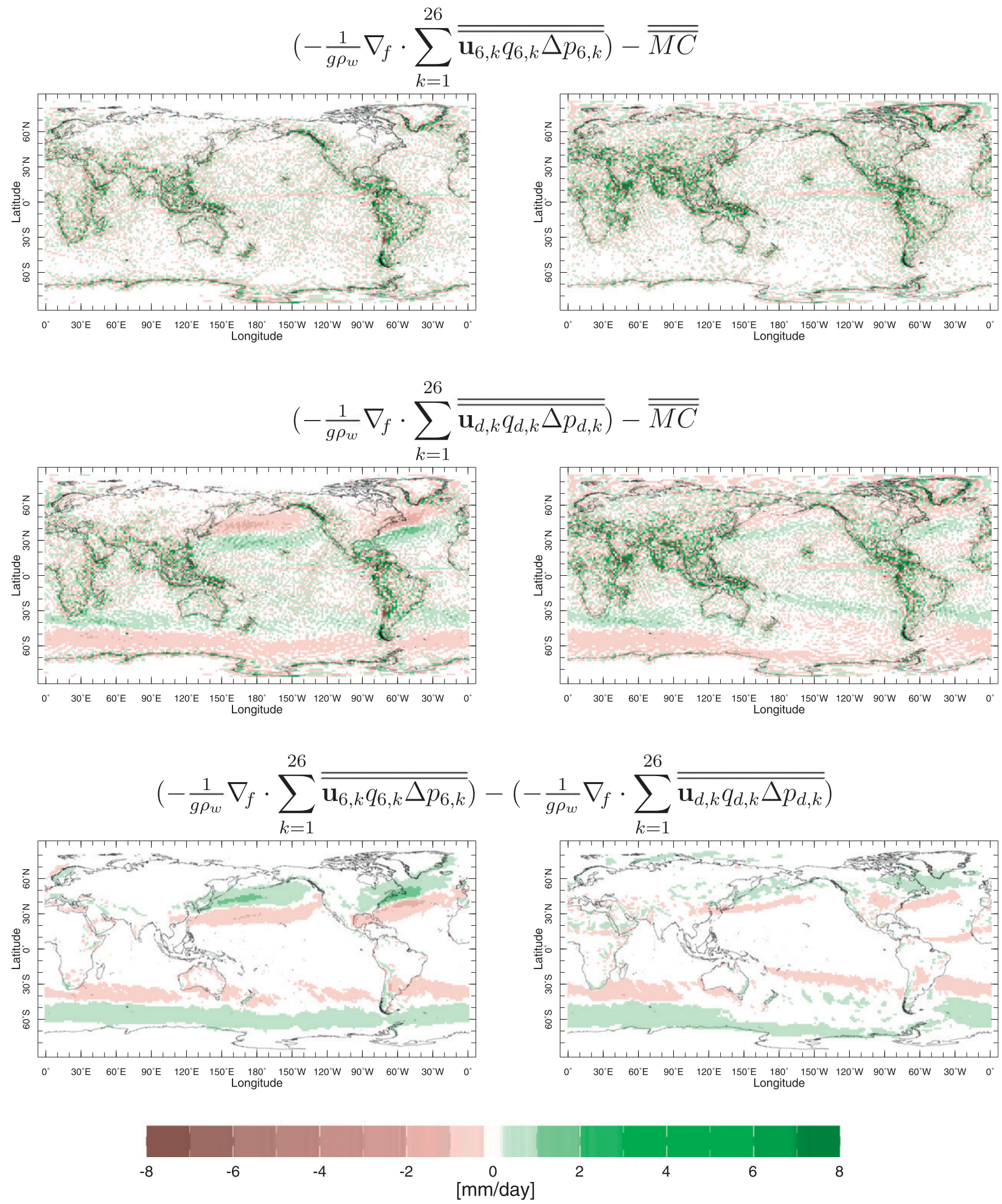

FIG. 3. The (left) January and (right) July climatological differences between the ERA-Interim-reported vertically integrated moisture convergence $\overline{\overline{\mathrm{MC}}}$ and that evaluated using (top) archived 6-hourly data on 26 pressure levels and (middle) daily data on 26 pressure levels. (bottom) The difference between 6-hourly and daily evaluations. (Units are $\mathrm{mm} \mathrm{day}^{-1}$.) 
TABLE 2. The long-term average of root-mean-square differences $\left(\mathrm{mm} \mathrm{day}^{-1}\right)$ between monthly mean diagnostically computed divergence of vertically integrated moisture content and the ERA-Interim-reported values of the same ( $\overline{\mathrm{MC}})$ for various combinations of vertical and time resolution of the diagnostic computations. Legend in the table corresponds to the usage in the main text except that $n$ generically refers to the time resolution, either 6 hourly or daily.

\begin{tabular}{|c|c|c|c|c|c|c|c|c|c|}
\hline \multicolumn{10}{|c|}{ Errors $\left(\mathrm{mm} \mathrm{day}^{-1}\right)$} \\
\hline & \multicolumn{3}{|c|}{26 levels } & \multicolumn{3}{|c|}{18 levels } & \multicolumn{3}{|c|}{8 levels } \\
\hline & $\overline{\overline{\mathbf{u}_{n} q_{n} \Delta p_{n}}}$ & $\overline{\overline{\mathbf{u}_{n} q_{n}}} \overline{\Delta p}$ & $\overline{\overline{\mathbf{u}_{n} q_{n}}} \overline{\overline{\Delta p}}$ & $\overline{\overline{\mathbf{u}_{n} q_{n} \Delta p_{n}}}$ & $\overline{\overline{\mathbf{u}_{n} q_{n}}} \overline{\Delta p}$ & $\overline{\overline{\mathbf{u}_{n} q_{n}}} \overline{\overline{\Delta p}}$ & $\overline{\overline{\mathbf{u}_{n} q_{n} \Delta p_{n}}}$ & $\overline{\overline{\mathbf{u}_{n} q_{n}}} \overline{\Delta p}$ & $\overline{\overline{\mathbf{u}_{n} q_{n}}} \overline{\overline{\Delta p}}$ \\
\hline \multicolumn{10}{|l|}{$6 \mathrm{~h}$} \\
\hline Global & 1.10 & 1.11 & 1.11 & 1.20 & 1.21 & 1.22 & 1.97 & 2.02 & 2.07 \\
\hline Land & 1.57 & 1.57 & 1.57 & 1.74 & 1.76 & 1.79 & 2.90 & 3.00 & 3.08 \\
\hline Ocean & 0.85 & 0.85 & 0.85 & 0.89 & 0.90 & 0.90 & 1.43 & 1.46 & 1.49 \\
\hline$\leq 30^{\circ} \mathrm{N} / \mathrm{S}$ & 1.06 & 1.06 & 1.06 & 1.12 & 1.12 & 1.12 & 1.81 & 1.83 & 1.86 \\
\hline $30^{\circ}-90^{\circ} \mathrm{N} / \mathrm{S}$ & 0.52 & 0.52 & 0.52 & 0.54 & 0.55 & 0.56 & 0.82 & 0.86 & 0.91 \\
\hline \multicolumn{10}{|l|}{ Daily } \\
\hline Global & 1.14 & 1.14 & 1.14 & 1.23 & 1.24 & 1.26 & 1.99 & 2.04 & 2.09 \\
\hline Land & 1.58 & 1.59 & 1.59 & 1.76 & 1.78 & 1.80 & 2.91 & 2.99 & 3.07 \\
\hline Ocean & 0.89 & 0.89 & 0.90 & 0.94 & 0.94 & 0.95 & 1.47 & 1.49 & 1.52 \\
\hline$\leq 30^{\circ} \mathrm{N} / \mathrm{S}$ & 1.07 & 1.07 & 1.07 & 1.13 & 1.13 & 1.13 & 1.82 & 1.84 & 1.86 \\
\hline $30^{\circ}-90^{\circ} \mathrm{N} / \mathrm{S}$ & 0.64 & 0.64 & 0.64 & 0.66 & 0.67 & 0.68 & 0.93 & 0.97 & 1.01 \\
\hline
\end{tabular}

and

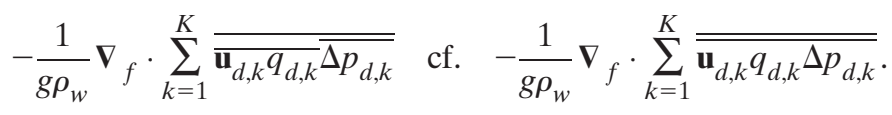

Figure 5 shows this comparison with daily data for both the 18- and 26-layer versions and with 6-hourly data for 26 layers. In no case are there important increases in error when going from daily vertical integrals to calculations that use monthly mean flow-humidity covariances together with monthly mean pressure thicknesses (see also Table 2). These comparisons show that no significant additional error is introduced by first time averaging the covariance of $\mathbf{u}$ and $q$ and then vertically integrating this using the time mean $p_{s}$ as the lower limit of integration.

\section{Breaking down the moisture budget into components related to divergent flow, mean flow advection of moisture and transient eddy fluxes}

The form of the moisture budget equation examined so far is quite useful and would allow a breakdown of, say, $P-E$ anomalies (or change) into components due to circulation and humidity anomalies (or change) since either $\mathbf{u}$ or $q$ can be held at climatological values while the other one is allowed to vary, all within the vertical integral and the divergence operator (see below). However, this form does not allow an assessment of the relative roles of divergent circulations (i.e., the $q \nabla \cdot \mathbf{u}$ term) and advection of moisture (i.e., the $\mathbf{u} \cdot \nabla q$ term) to $P-E$. To assess that, we must return to a form with the divergence operator inside the vertical integral, which then introduces the surface boundary term as in Eqs. (14) and (15). The problem then emerges when trying to evaluate the $\int_{0}^{p_{s}} \nabla \cdot(\mathbf{u} q) d p$ term because, in the presence of varying surface pressure, the lower limit of integration is different at the grid points used to perform the divergence operator. For example, is the right approach to evaluate $\boldsymbol{\nabla} \cdot(\mathbf{u} q) \approx \nabla_{f} \cdot(\mathbf{u} q)$ only at the pressure levels that exist for all the points used in the divergence operator [Eq. (15)], $(i+1, j),(i-1, j)$, $(i, j+1)$, and $(i, j-1)$, or is the right approach to also incorporate grid points that are at pressure levels which are nonexistent (higher pressure than surface pressure) and assume that $\mathbf{u}$ is zero at those points? In either case, how is the surface boundary term to be evaluated?

Fortunately, there is a way to do this that yields the correct answer. To illustrate the approach we will reduce the problem to $(x, p)$ dimensions and examine

$$
\frac{\partial}{\partial x}\left(\int_{0}^{p_{s}}(u q) d p\right)=\int_{0}^{p_{s}} \frac{\partial(u q)}{\partial x} d p+u_{s} q_{s} \frac{\partial p_{s}}{\partial x}
$$

where $x=a \lambda \cos \phi$ and require that the numerical methods chosen to evaluate these terms ensure a balance.

Referring to Fig. 6 and temporarily reintroducing $i$ subscripts on $K$, we use $K_{i}$ to indicate the lowest pressure 


\section{January climatology July climatology}

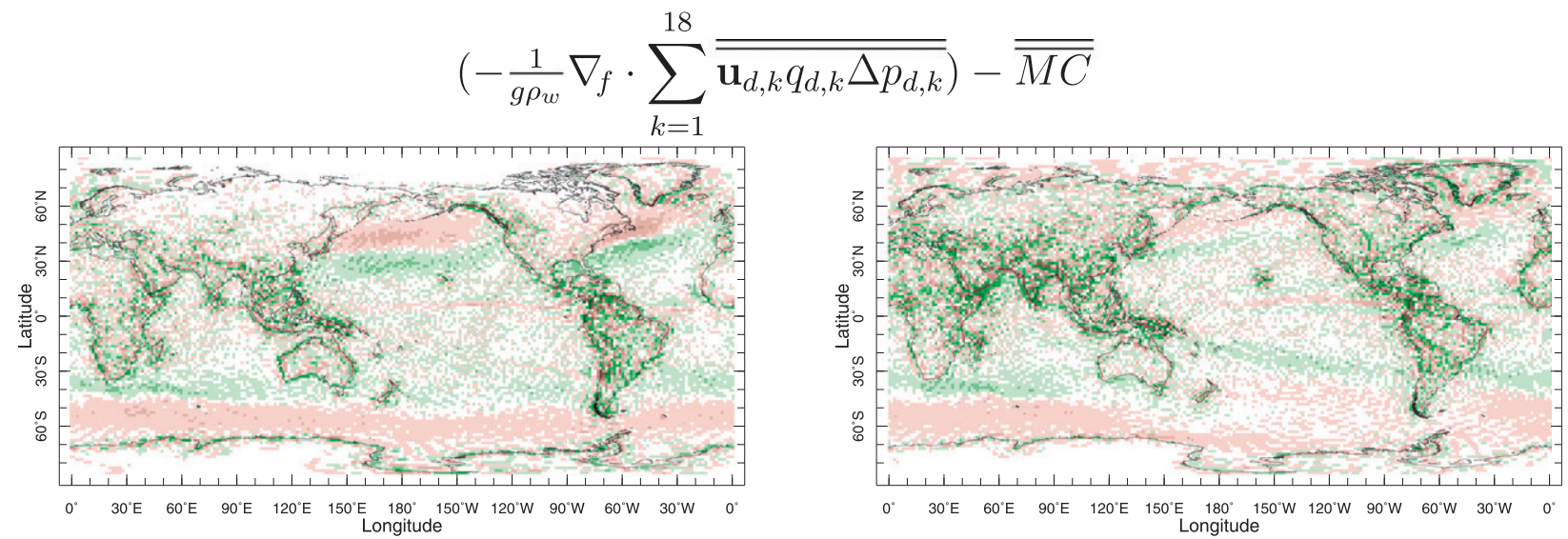

$$
\left(-\frac{1}{g \rho_{w}} \nabla_{f} \cdot \sum_{k=1}^{8} \overline{\overline{\mathbf{u}_{d, k} q_{d, k} \Delta p_{d, k}}}\right)-\overline{\overline{M C}}
$$
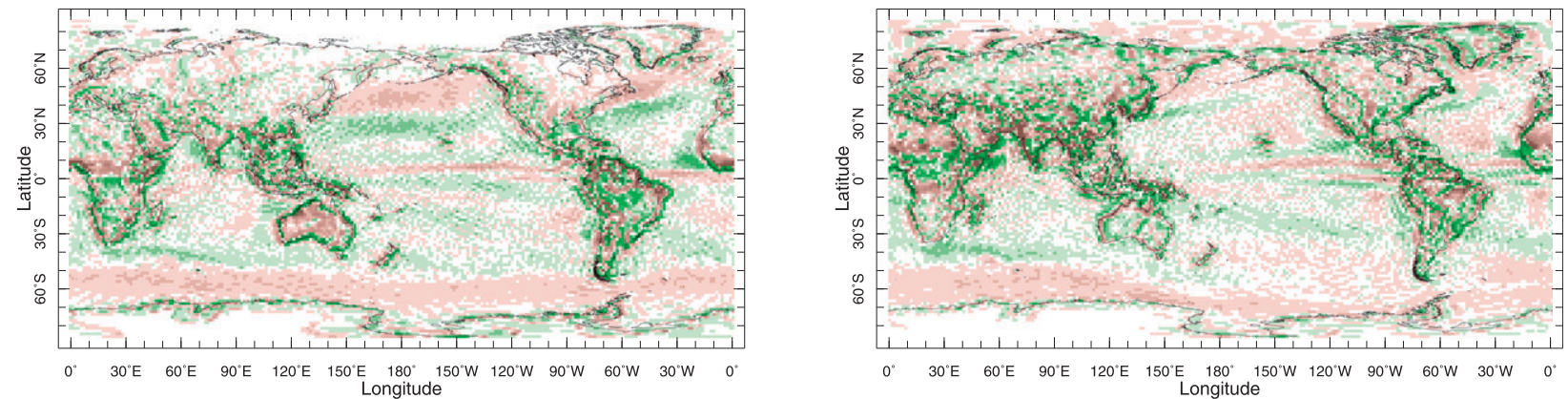

$$
\left(-\frac{1}{g \rho_{w}} \nabla_{f} \cdot \sum_{k=1}^{26} \overline{\overline{\mathbf{u}_{d, k} q_{d, k} \Delta p_{d, k}}}\right)-\left(-\frac{1}{g \rho_{w}} \nabla_{f} \cdot \sum_{k=1}^{18} \overline{\overline{\mathbf{u}_{d, k} q_{d, k} \Delta p_{d, k}}}\right)
$$
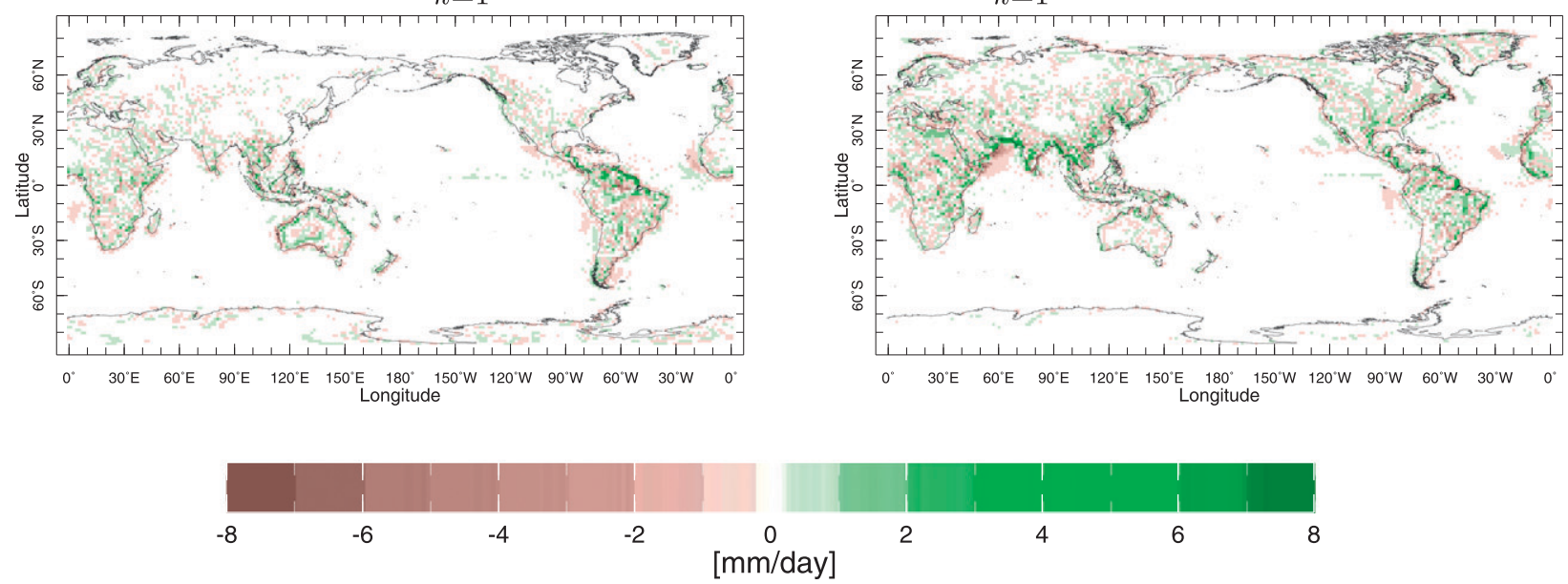

FIG. 4. The (left) January and (right) July climatological differences between the ERA-Interim-reported vertically integrated moisture convergence $\overline{\overline{\mathrm{MC}}}$ and that evaluated using (top) archived daily data on 18 pressure levels and (middle) 8 pressure levels. (bottom) The difference between evaluations using daily data and 26 levels vs 18 levels. (Units are $\mathrm{mm} \mathrm{day}^{-1}$.) 
January climatology July climatology
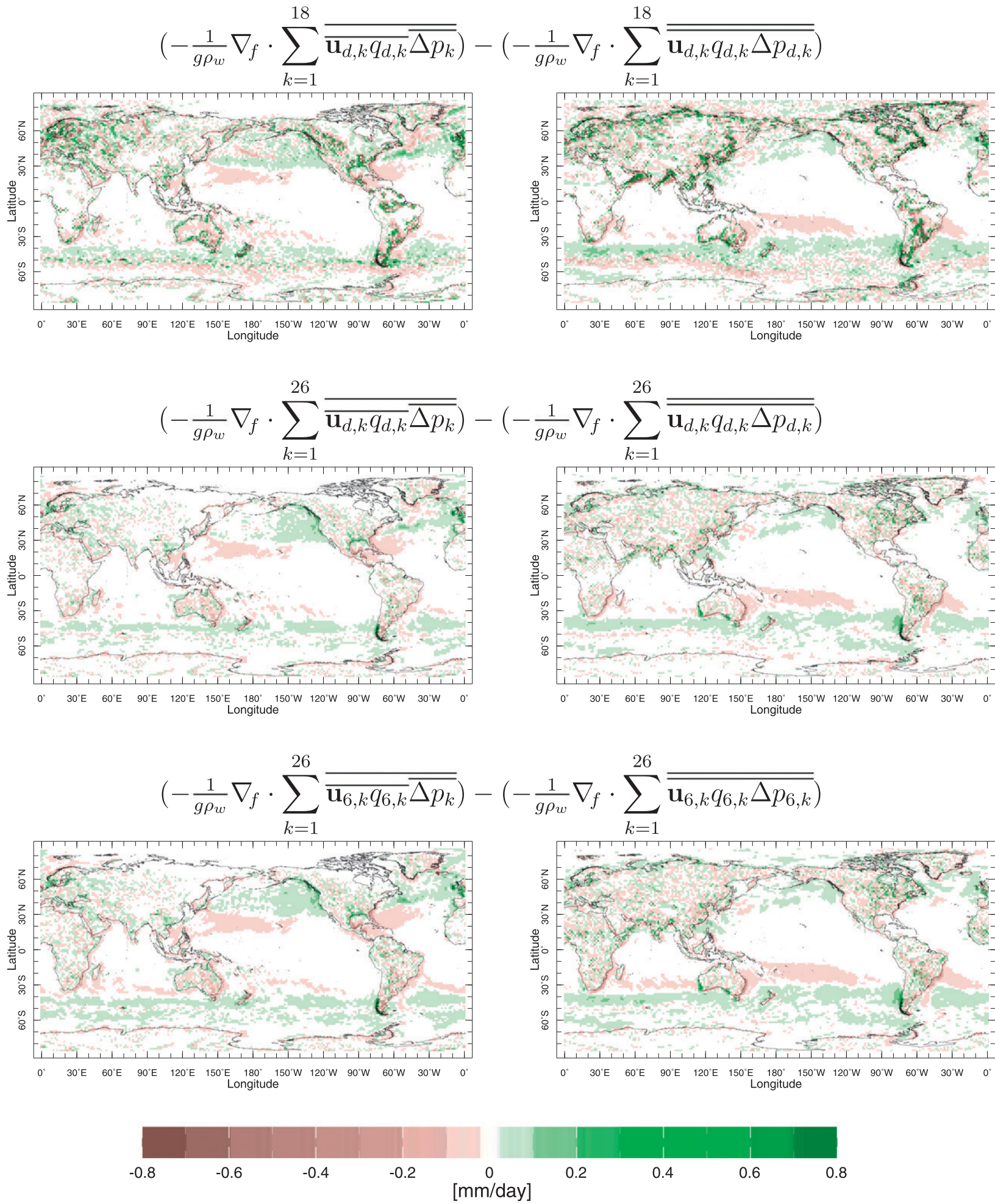

FIG. 5. The (left) January and (right) July climatological differences between evaluations of the convergence of vertically integrated moisture for the cases of using monthly means of daily wind and humidity covariances combined with monthly mean pressure thicknesses and the case that allows for daily covariances of wind, humidity, and pressure thicknesses with (top) 18 and (middle) 26 pressure levels and (bottom) the same difference using 26 pressure levels and 6-hourly data. (Units are mm day ${ }^{-1}$.) 
level at grid point $i$ that is above the surface: that is, has a pressure, $p_{K_{i}}$ lower than the surface pressure at the grid point $p_{s_{i}}$. Then Eq. (21), evaluated between grid points $i$ and $i+1$, is approximated by

$$
\begin{aligned}
{\left[\frac{\partial}{\partial x}\left(\int_{0}^{p_{s}}(u q) d p\right)\right]_{i+1 / 2} \approx } & \frac{1}{x_{i+1}-x_{i}}\left\{\sum_{k=1}^{K_{i+1}}(u q)_{i+1, k} \Delta p_{k}+(u q)_{i+1, K_{i+1}}\left(p_{s, i+1}-p_{K+1 / 2}\right)\right. \\
& \left.-\left[\sum_{k=1}^{K_{i}}(u q)_{i, k} \Delta p_{k}+(u q)_{i, K_{i}}\left(p_{s, i}-p_{K+1 / 2}\right)\right]\right\} .
\end{aligned}
$$

Here, for example, at a latitude $\phi, x_{i}=a \cos \phi \lambda_{i}$. Next we let the level $k=\mathrm{kk}$ equal the lowest level with pressure $p=p_{\mathrm{kk}}$ for which all the adjacent grid points have $p_{s} \geq$ $p_{\text {kk }}$. Then Eq. (22) can be rewritten as

$$
\begin{aligned}
{\left[\frac{\partial}{\partial x}\left(\int_{0}^{p_{s}}(u q) d p\right)\right]_{i+1 / 2} \approx } & \frac{1}{x_{i+1}-x_{i}}\left\{\sum_{k=1}^{\mathrm{kk}}\left[(u q)_{i+1, k}-(u q)_{i, k}\right] \Delta p_{k}+\sum_{k=\mathrm{kk}+1}^{K_{i+1}}(u q)_{i+1, k} \Delta p_{k}\right. \\
& \left.-\sum_{k=\mathrm{kk}+1}^{K_{i}}(u q)_{i, k} \Delta p_{k}+(u q)_{i+1, K_{i+1}}\left(p_{s, i+1}-p_{K+1 / 2}\right)-(u q)_{i, K_{i}}\left(p_{s, i}-p_{K+1 / 2}\right)\right\},
\end{aligned}
$$

where it is understood that the sum $\sum_{k=\mathrm{kk}+1}^{K}$ is only performed for $K \geq \mathrm{kk}+1$, which by definition means only at $i+1$ for surface height decreasing westward and $i$ for surface height increasing westward.

The first right-hand side term in Eq. (23) provides a straightforward approximation to the first right-hand side term in Eq. (21): namely,

$$
\left[\int_{0}^{p_{s}} \frac{\partial(u q)}{\partial x} d p\right]_{i+1 / 2} \approx \sum_{k=1}^{\mathrm{kk}} \frac{(u q)_{i+1, k}-(u q)_{i, k}}{x_{i+1}-x_{i}} \Delta p_{k}
$$

The remainder of Eq. (22) provides an approximation to the surface term in Eq. (20) as follows:

$$
\begin{aligned}
\left(u_{s} q_{s} \frac{\partial p_{s}}{\partial x}\right)_{i+1 / 2}= & \frac{1}{x_{i+1}-x_{i}}\left\{\sum_{k=\mathrm{kk}+1}^{K_{i+1}}(u q)_{i+1, k} \Delta p_{k}-\sum_{k=\mathrm{kk}+1}^{K_{i}}(u q)_{i, k} \Delta p_{k}\right. \\
& \left.+(u q)_{i+1, K_{i+1}}\left(p_{s, i+1}-p_{K+1 / 2}\right)-(u q)_{i, K_{i}}\left(p_{s, i}-p_{K+1 / 2}\right)\right\} .
\end{aligned}
$$

We refer to this surface term as $\mathrm{SFC}_{K}$. The fact that this approximation holds can be seen by supposing the special case when $u q$ is uniform everywhere and hence equals $\left(u_{s} q_{s}\right)_{i+1 / 2}$ in which case Eq. (25) reduces to

$$
\left(u_{s} q_{s} \frac{\partial p_{s}}{\partial x}\right)_{i+1 / 2}=\left(u_{s} q_{s}\right)_{i+1 / 2} \frac{p_{s, i+1}-p_{s, i}}{x_{i+1}-x_{i}} .
$$

If the surface term is evaluated as in Eq. (25) and the vertical integral of the divergence of moisture as in Eq. (24), then the sum of these two terms will exactly equal that given by Eq. (22) [or Eq. (23)] and the balance in Eq. (21) is assured. As such, since all the data needed to evaluate both Eqs. (22) and (24) are typically available, we would recommend that the surface term be evaluated as the difference between these and avoid the need to explicitly calculate it from Eq. (25).
It should be noted that the surface term, despite not being easily interpreted in a physical way, is not small. In Fig. 7 we show the annual mean climatological moisture budget terms. Comparison of the mean flow moisture convergence (top right) with the total moisture convergence (top left) shows how dominant the mean flow is in explaining the moisture budget while the differences show the importance of the transient eddies in the midlatitudes and subtropics. Figure 7 also shows the vertical integral of moisture divergence [the two-dimensional analog of Eq. (24)] and the surface term the two-dimensional analog of Eq. (25) but evaluated as a residual between two-dimensional analogs of Eqs. (23) [or Eq. (22)] and (24)\}. It is clear that, for the moisture transport by the mean flow, the pattern and amplitude is preserved whether the convergence is computed before 


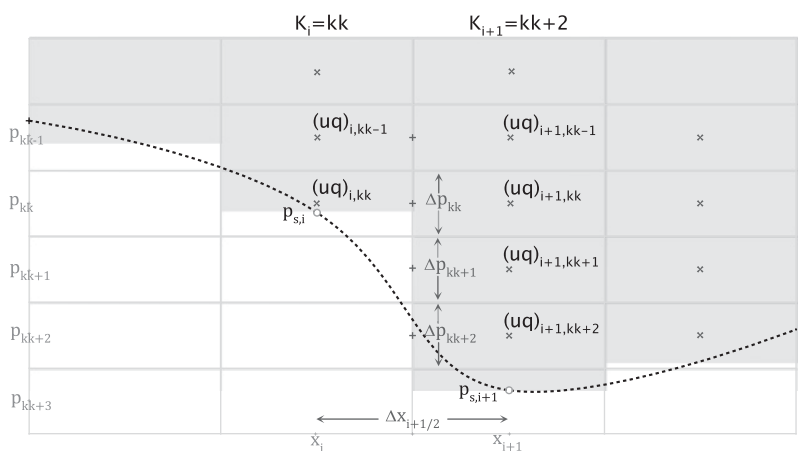

FIG. 6. Schematic of a pressure grid over uneven topography for reference in discussion of how to evaluate the surface term that appears when evaluating vertical integrals of moisture divergence: that is, when the divergence operator is inside the vertical integral over pressure. The terms $K_{i}$ and $K_{I+1}$ indicate number of vertical pressure levels at columns $i$ and $i+1$ and kk indicates the lowest level for which the pressure $p_{k}$ is lower than the surface pressure at both grid points, $i$ and $i+1$, needed to evaluate the divergence operator at $i+1 / 2$.

or after the vertical integral is performed. However, it is also clear that the surface term $\overline{\mathrm{SFC}}_{K}$ is large wherever there are large gradients of surface pressure such as at coasts (where altitude can change abruptly) and over mountain ranges and hence cannot be ignored in the moisture budget.

Bringing the divergence operator inside the vertical integral allows the moisture divergence term to be broken into components related to the divergent flow and to advection across humidity gradients as in Eq. (15). This is usually performed on the monthly mean fields. Denoting once more the ERA-reported monthly means by a single overbar, in Fig. 7 we also show climatological values of the terms in

$$
\begin{aligned}
-\frac{1}{g \rho_{w}} \sum_{k=1}^{\mathrm{kk}} \overline{\nabla_{f} \cdot\left(\overline{\mathbf{u}}_{k} \bar{q}_{k}\right) \overline{\Delta p}_{k}}= & -\frac{1}{g \rho_{w}} \sum_{k=1}^{\mathrm{kk}} \overline{\left(\bar{q}_{k} \nabla_{f} \cdot \overline{\mathbf{u}}_{k}\right) \overline{\Delta p}_{k}} \\
& -\frac{1}{g \rho_{w}} \sum_{k=1}^{\mathrm{kk}} \overline{\left(\overline{\mathbf{u}} \cdot \nabla_{f} \bar{q}_{k}\right) \overline{\Delta p}_{k}} .
\end{aligned}
$$

The mass divergence is clearly the dominant term in explaining the pattern of the mean flow moisture divergence. However, the mean flow advection term acts to dry the tropics, where the trades flow from drier regions to moister regions and moistens the midlatitudes, where the surface westerlies flow from moister regions to drier regions.

\section{Summary}

Table 2 provides a quantitative assessment of the sizes of the various sources of error. First of all we see that errors are much larger over land than ocean, presumably due to the complexity of three-dimensional spatial structures of winds and humidity. Errors are also larger in the tropics than extratropics, but this follows from the moisture convergences and divergences being larger there. The increase in error going from 6-hourly to daily data is, however, concentrated in the extratropics and is related to the transient eddy moisture transport. Errors due to reduced vertical resolution are not striking in going from 26 to 18 levels but are large over land and ocean, in the tropics and extratropics, when going to only 8 levels (typical of CMIP archives of daily data). Using monthly mean flow-humidity covariances together with monthly mean pressure thicknesses is in all cases an acceptable approximation.

\section{Errors in the evaluation of moisture budget anomalies: Case study of the 1993 Mississippi valley flood}

We have demonstrated the errors that are introduced into moisture budgets when evaluated diagnostically with archived data. However, that was done with climatological moisture budgets. Next we need to assess the errors involved when analyzing the moisture budget anomalies associated with certain events of interest such as floods and droughts. It is possible, after all, that the climatological errors are persistent enough in time that they do not appear within the anomalous budgets. To examine this, we choose the case of the late spring-early summer 1993 Mississippi valley flood, which represents an extreme seasonal anomaly of $P-E$ sustained by anomalous moisture convergence.

The analysis was conducted with the 26-level and 6-hourly data but using integration down to the monthly mean (as opposed to daily) surface pressure since we showed in section 4 that this approximation does not introduce important error. The equation we begin with is then

$$
\overline{(P-E)}_{\mathrm{der}}=-\frac{1}{g \rho_{w}} \nabla_{f} \cdot \sum_{k=1}^{K} \overline{\mathbf{u}_{6, k} q_{6, k}} \overline{\Delta p_{6, k}} .
$$

Here, as before, the single overbar denotes monthly mean quantities and $\overline{(P-E)}_{\text {der }}$ indicates the $P-E$ implied by the evaluated moisture convergence (as opposed to that reported by ERA-Interim or implied by $\overline{\mathrm{MC}})$. We are interested in evaluating this for the average of May-July (MJJ) 1993, when the floods occurred, and determining the anomalies relative to the climatological situation. With ERA-Interim we 


\section{Annual Climatology}

$$
-\frac{1}{g \rho_{w}} \nabla_{f} \cdot \sum_{k=1}^{26} \overline{\overline{\mathbf{u}_{6, k} q_{6, k}}} \overline{\Delta p_{k}}
$$

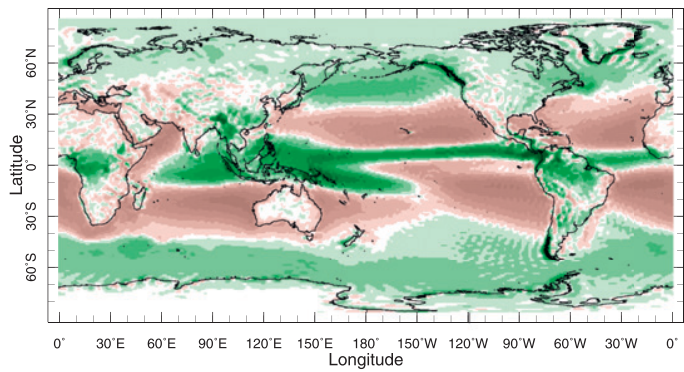

$$
-\frac{1}{g \rho_{w}} \sum_{k=1}^{26} \overline{\nabla_{f} \cdot \overline{\mathbf{u}_{6, k} q_{6, k}} \overline{\Delta p_{k}}}
$$

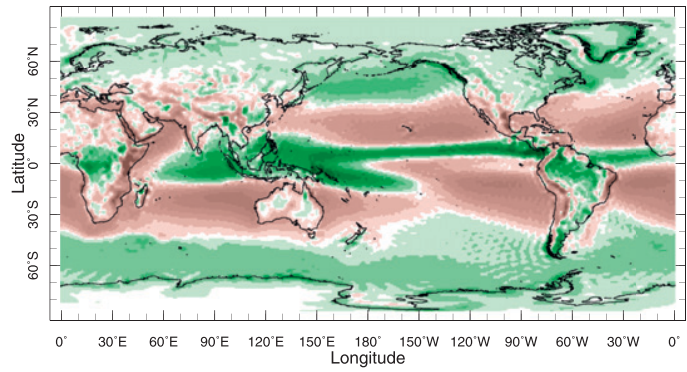

$\overline{\overline{S F C_{K}}}$
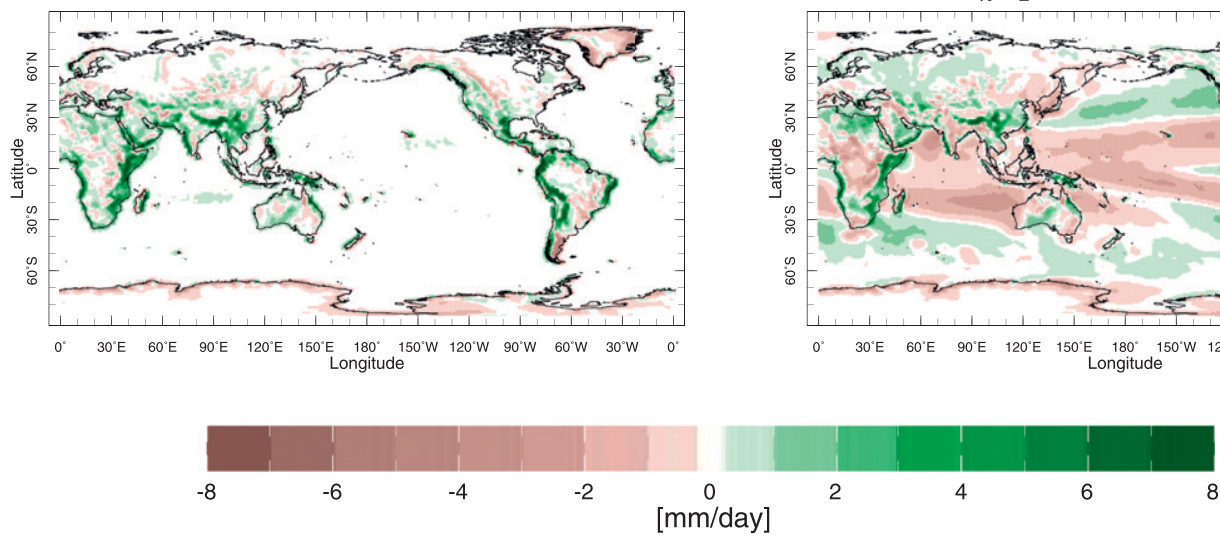

FIG. 7. (top left) The annual mean climatology of the convergence of vertically integrated total moisture flux and its two components, (middle left) the vertical integral of total moisture convergence and (bottom left) the total surface term. (top right) The convergence of vertically integrated mean flow moisture flux is split into components due to the (middle right) convergence mean flow and (bottom right) mean flow advection. (Units are $\mathrm{mm} \mathrm{day}^{-1}$.)

can evaluate the moisture convergence anomalies for MJJ 1993 directly from the reported values of MC and then we can also evaluate this from Eq. (28). Therefore, using the second overbar to denote the long-term climatological monthly mean and a hat above an overbar to denote a departure of a particular monthly mean from the climatological value (e.g., $\bar{q}=\overline{\bar{q}}+\widehat{\bar{q}}$ ), we evaluate 


$$
\widehat{\overline{\mathrm{MC}}}=\overline{\mathrm{MC}}-\overline{\overline{\mathrm{MC}}}
$$

and

$$
\begin{aligned}
& -\frac{1}{g \rho_{w}} \nabla_{f} \cdot \sum_{k=1}^{K} \overline{\overline{\mathbf{u}_{6, k} q_{6, k}} \overline{\Delta p_{6, k}}}=-\frac{1}{g \rho_{w}} \nabla_{f} \cdot \sum_{k=1}^{K} \overline{\mathbf{u}_{6, k} q_{6, k}} \overline{\Delta p_{6, k}} \\
& -\left(-\frac{1}{g \rho_{w}} \nabla_{f} \cdot \sum_{k=1}^{K} \overline{\overline{\mathbf{u}_{6, k} q_{6, k}}} \overline{\overline{\Delta p_{6, k}}}\right) .
\end{aligned}
$$

In Fig. 8 we show for MJJ 1993 (i.e., the average of the anomalies for the 3 months) the ERA-Interim-reported vertically integrated moisture convergence anomaly $\widehat{\mathrm{MC}}$, the estimate of this using 6-hourly archived data on 26 levels [i.e., the left-hand side of Eq. (30)] for both the globe and North America, the ERA-Interim-reported $\overline{P-E}$, and the change of vertically integrated moisture across the 3-month period. Globally, there is a close level of agreement between the actual column integrated moisture convergence anomaly and that diagnostically calculated with the largest anomalies being moisture convergence over the central and western equatorial Pacific and divergence to the north and south and within the Pacific ITCZ, consistent with outgoing longwave radiation anomalies at the time and related to a waning El Niño (e.g., Trenberth and Guillemot 1996). Over North America the agreement is also good and shows a large and focused moisture convergence anomaly over the upper Mississippi valley and a moisture divergence anomaly over most of the southern United States and the western Atlantic Ocean. The ERA-Interim-reported $\widehat{\overline{P-E}}$ anomaly over North America agrees quite well with $\widehat{\mathrm{MC}}$. The change in moisture storage is small.

To assess the level of agreement between the actual and diagnostically computed anomalies, in Fig. 9 we show the differences between ERA-Interim-reported and diagnostically computed column integrated moisture convergence for MJJ 1993 and, for comparison, the climatological MJJ. The climatological error in MJJ is similar in character to that in the other seasons (Fig. 1) and is noisy and not systematic over North America. The MJJ 1993 error is also not systematic and also smaller than the climatological difference. This means that the anomalous moisture convergence in any one month, season, or-presumably-year can indeed be estimated in a useful way by the diagnostic computation. That this is so allows further analysis of dynamical and thermodynamical causes of the anomalies of interest.

To determine causes of $P-E$ anomalies, we break down the moisture convergence anomaly into components due to mean circulation anomalies, mean humidity anomalies, and transient eddy moisture flux anomalies.
To do this we first note that 6-hourly quantities are given, for example, for $q_{6}$, by

$$
q_{6}=\bar{q}+q_{6}^{\prime}=\overline{\bar{q}}+\widehat{\bar{q}}+q_{6}^{\prime},
$$

where the prime denotes a departure of 6-hourly data from the monthly mean (which itself equals the climatological monthly mean plus the monthly mean anomaly). Substituting expansions like Eq. (31) into Eq. (28), we can derive equations for the monthly mean climatology and anomalies in $\overline{(P-E)_{\text {der }}}$ or equivalently the diagnostically computed moisture convergence, in terms of components of the flow and humidity fields,

$$
\begin{aligned}
& \overline{\overline{(P-E)}})_{\mathrm{der}} \approx-\frac{1}{g \rho_{w}} \nabla_{f} \cdot \sum_{k=1}^{K}\left(\overline{\overline{\mathbf{u}_{k}}} \overline{\overline{q_{k}}}+\overline{\overline{u_{6, k}^{\prime} q_{6, k}^{\prime}}}\right) \overline{\overline{\Delta p_{k}}} \\
& \left.(\overline{\overline{P-E}})_{\mathrm{der}} \approx-\frac{1}{g \rho_{w}} \nabla_{f} \cdot \sum_{k=1}^{K} \overline{\left(\overline{\mathbf{u}}_{k} \bar{q}_{k} \overline{\Delta p_{k}}\right.}+\overline{\overline{\mathbf{u}_{6, k}^{\prime} q_{6, k}^{\prime}} \overline{\Delta p_{k}}}\right) \\
& \approx-\frac{1}{g \rho_{w}} \nabla_{f} \cdot \sum_{k=1}^{K}\left(\overline{\overline{\mathbf{u}}}_{k} \widehat{\bar{q}}_{k}+\widehat{\overline{\mathbf{u}}}_{k} \overline{\bar{q}}_{k}+\overline{\overline{\mathbf{u}_{6, k}^{\prime} q_{6, k}^{\prime}}}\right) \overline{\overline{\Delta p_{k}}}
\end{aligned}
$$

where, to derive the approximation in Eq. (34), products of monthly anomalies and terms involving $\overline{\Delta p_{k}}$ have been neglected. [It was found that, in general, ignoring the surface pressure variations which dictate variations in $\overline{\Delta p_{k}}$ introduces little additional error. Further, in the case of Eq. (34), which combines terms that are climatological and terms that are monthly anomalies, it would be ambiguous what to use for $\overline{\Delta p_{k}}$; hence, using climatological values seems expedient. $\left.{ }^{1}\right]$ In Eq. (34), the first term on the right-hand side is the anomaly in implied $P-E$ due to anomalies in mean specific humidity working with the climatological circulation, the second term is the anomaly due to the anomaly in mean circulation working with the climatological specific humidity, and the third term is the anomaly due to anomalies in the moisture convergence by submonthly time-scale transient eddies.

In Fig. 10 we show the combined contribution of the mean flow and mean humidity to the moisture convergence anomaly and also the contribution from transient

\footnotetext{
${ }^{1}$ In Seager et al. (2012) (see also Seager and Naik 2012), anomalies in moisture budgets were examined using compositing over model El Niño and La Niña events and the pressure integrals were chosen to correspond to surface pressure anomalies during these events, but the ambiguity introduced by breakdowns into terms combining climatological and anomaly quantities is not avoided.
} 
MJJ 1993
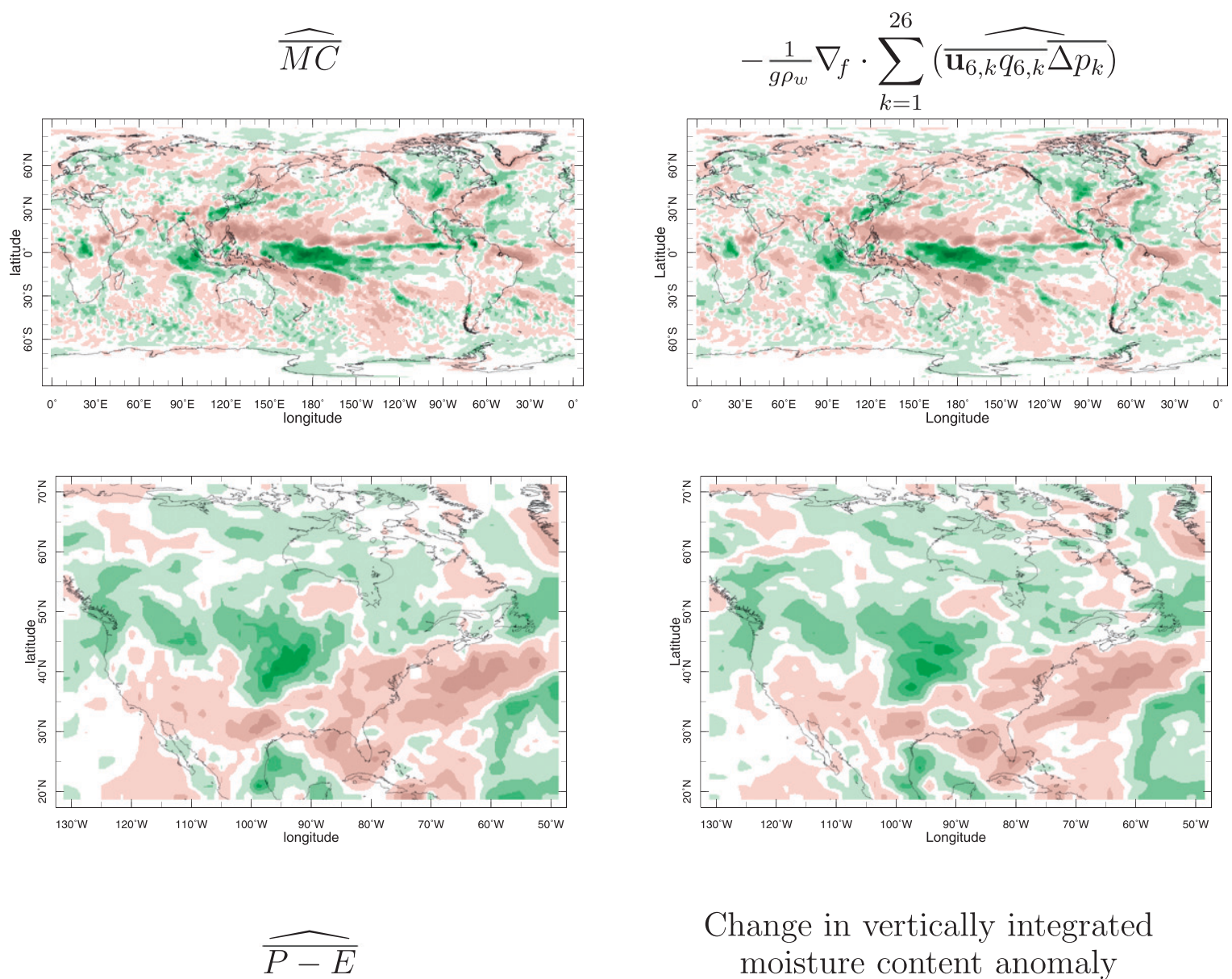

Change in vertically integrated moisture content anomaly
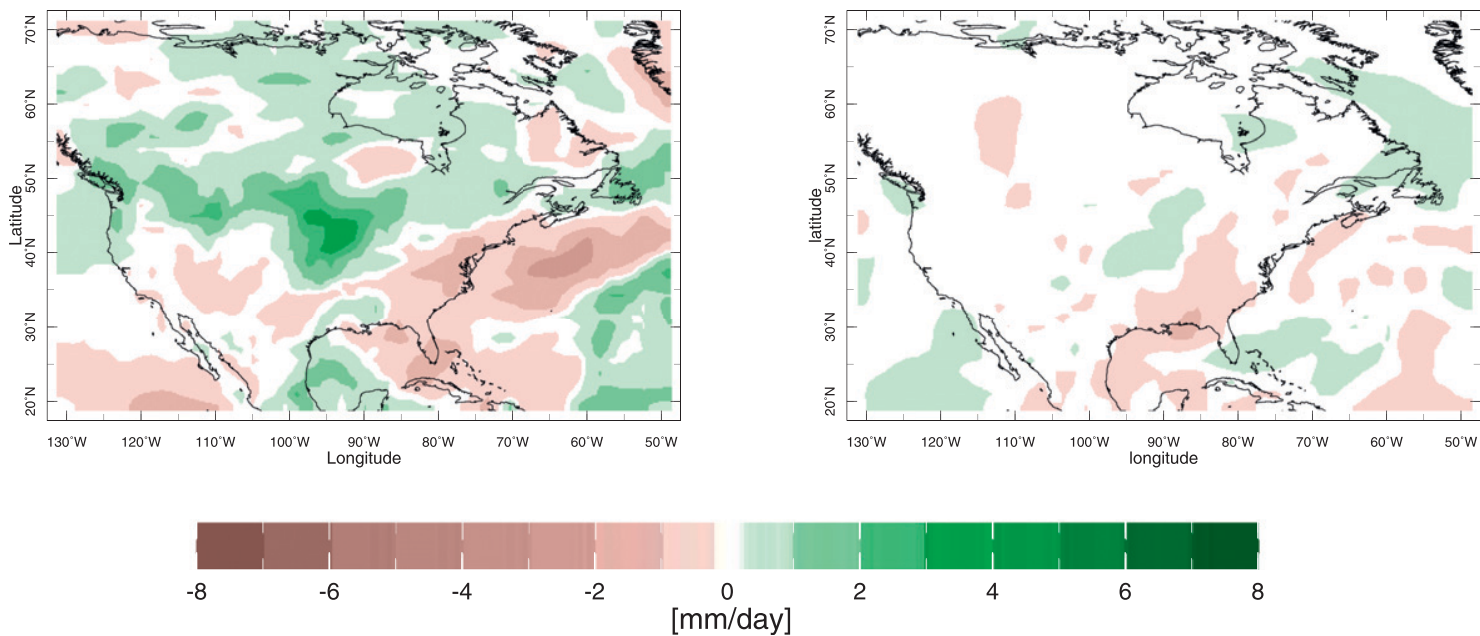

FIG. 8. (top left),(middle left) The ERA-Interim-reported vertically integrated moisture convergence anomaly and (top right),(middle right) that computed diagnostically from 6-hourly data on 26 levels for May-July 1993 for (top) the globe and (middle) North America. (bottom left) The ERA-Interim-reported $\widehat{\overline{P-E}}$ and (bottom right) the change in moisture storage. (Units are mm day ${ }^{-1}$.) 


\section{MJJ climatological error}

$$
\overline{\overline{M C}}-\left(-\frac{1}{g \rho_{w}} \nabla_{f} \cdot \sum_{k=1}^{26} \overline{\left(\overline{\mathbf{u}_{6, k} q_{6, k}} \overline{\Delta p_{k}}\right)}\right)
$$
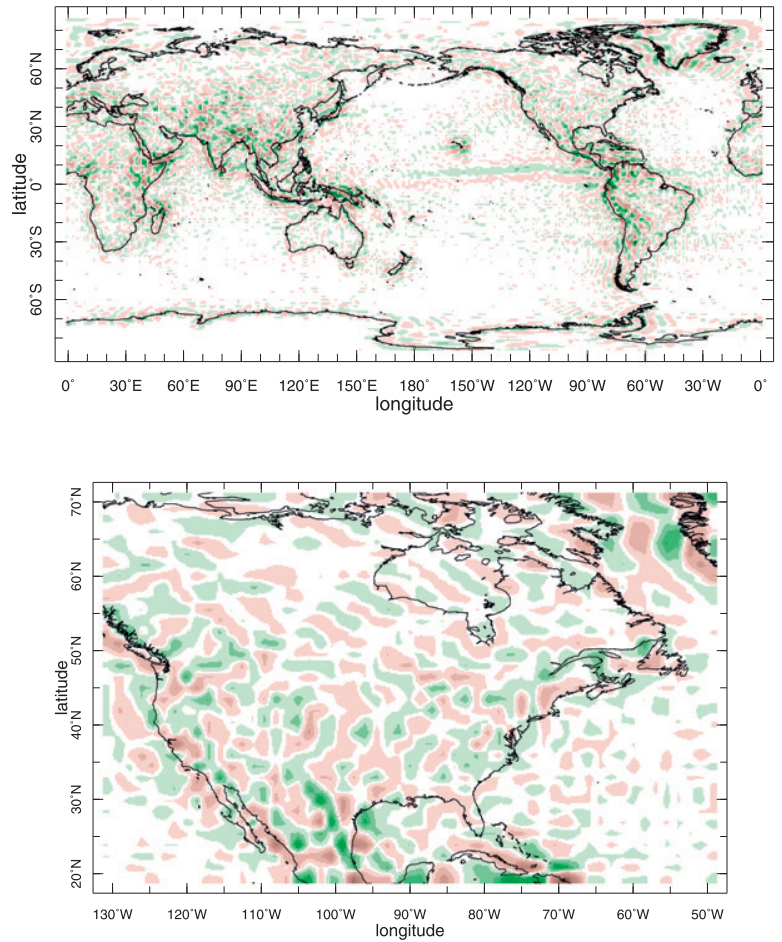

MJJ 1993 error
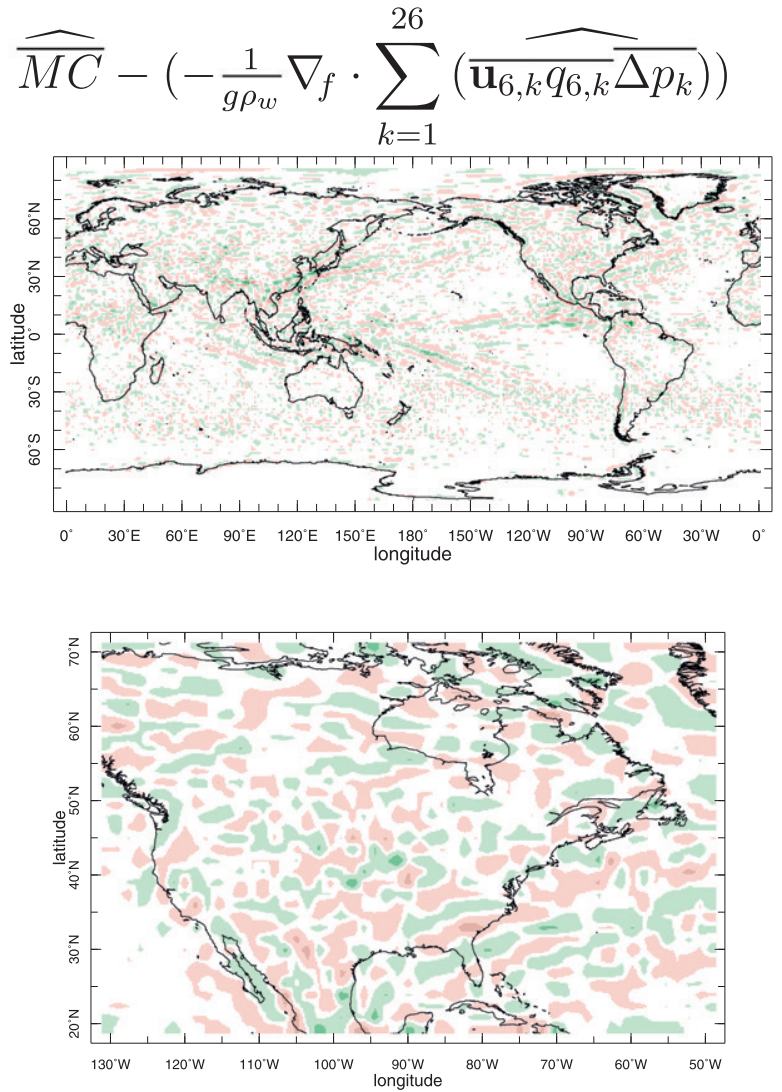

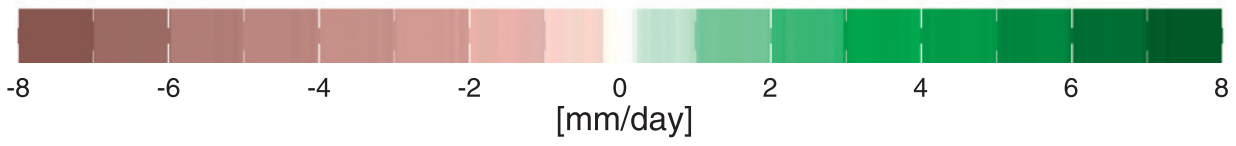

FIG. 9. The difference between ERA-Interim-reported vertically integrated moisture convergence and that computed diagnostically with 6-hourly data on 26 levels (left) for climatological MJJ and (right) just for MJJ 1993. (Units are mm day ${ }^{-1}$.)

eddy moisture convergence, using now combinations of 18 and 26 levels and 6-hourly and daily data [i.e., the breakdown in Eq. (33)]. The mean flow and humidity anomalies caused the moisture convergence anomaly in the central United States, and this is well approximated with only 18 levels. The contribution of mean flow moisture convergence to the floods is consistent with the persistently strong Great Plains low-level jet identified by Weaver et al. (2009). The transient eddy moisture convergence anomaly, in contrast, provides a northsouth dipole with divergence over the southeastern United States and convergence to the north resulting in a shift northward of the total moisture convergence anomaly. The transient eddy moisture convergence anomaly evaluated with 6-hourly data is well approximated with 18 levels. The transient eddy moisture flux convergence pattern is consistent with the argument of
Trenberth and Guillemot (1996) (based on flux anomalies but not on convergence) that the storm-track anomalies in MJJ 1993 transferred moisture from the Gulf of Mexico into the upper Mississippi valley. When the transient eddy moisture convergence and divergence anomalies are evaluated with daily data the patterns are consistent with their 6-hourly counterparts but are notably weaker. As for the climatological case, it is clear that daily data is inadequate for evaluating transient eddy fluxes and divergence and that accuracy requires 6-hourly data.

The next step is to determine the relative contribution to the $P-E$ anomaly of changes in the mean flow and changes in the mean humidity: that is, the breakdown in Eq. (34). In Fig. 11 we show the mean flow moisture convergence anomaly (repeated from Fig. 10), together with the anomalous mean moisture flux vectors and then the part of this that is caused by the flow anomalies 
MJJ 1993
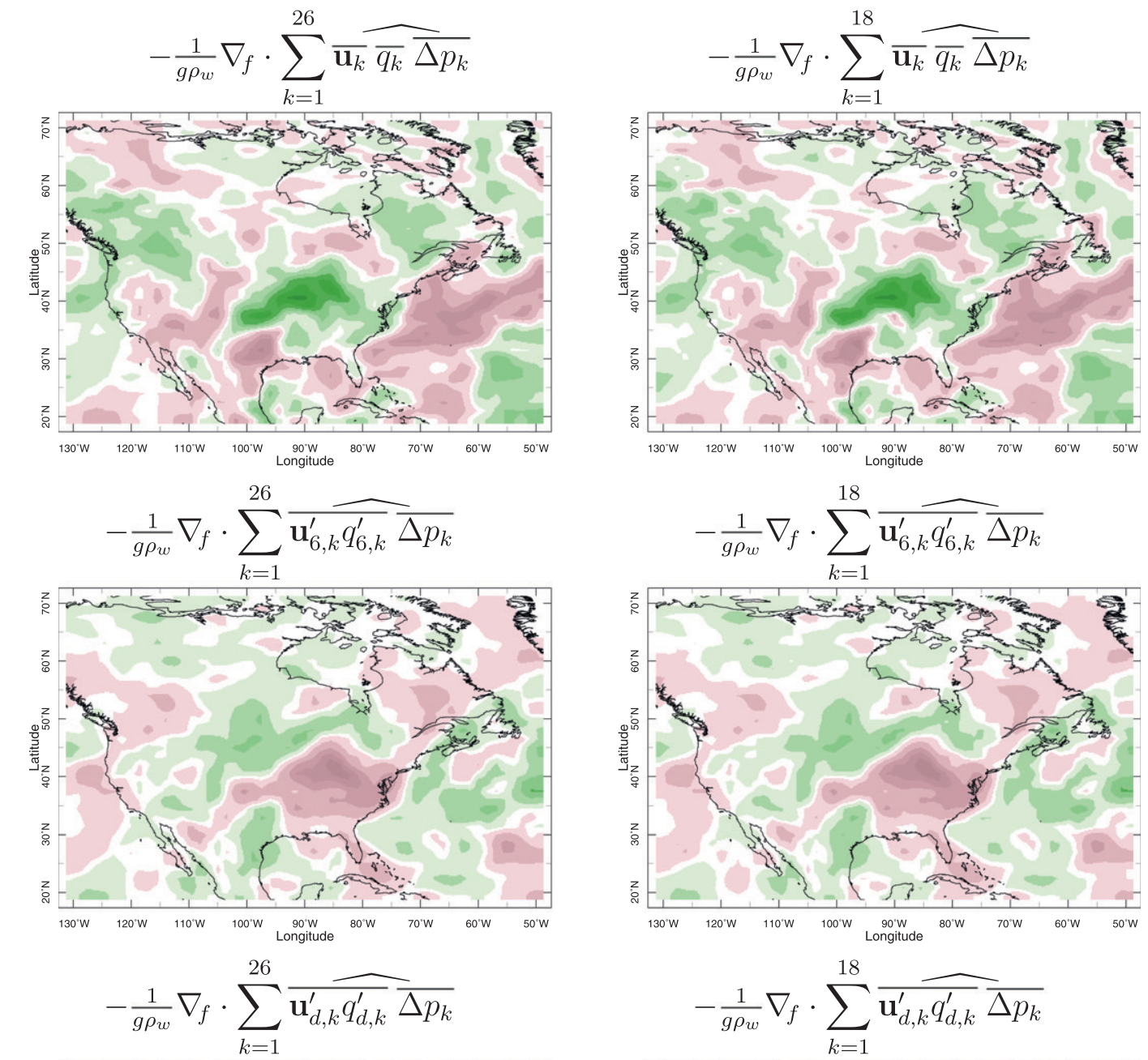

$$
-\frac{1}{g \rho_{w}} \nabla_{f} \cdot \sum_{k=1}^{18} \widehat{\widehat{\mathbf{u}_{6, k}^{\prime} q_{6, k}^{\prime}} \overline{\Delta p_{k}}}
$$
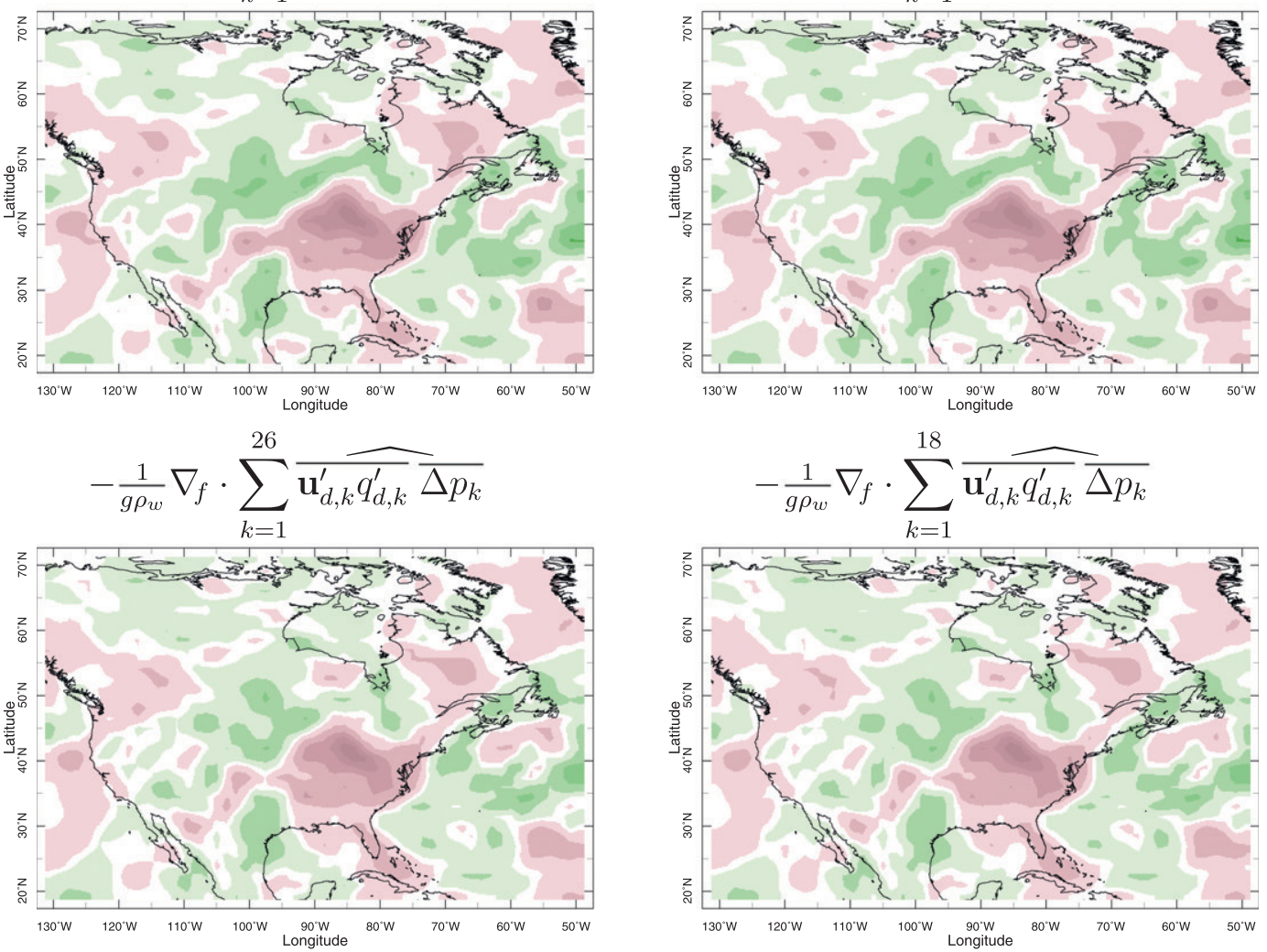

$$
-\frac{1}{g \rho_{w}} \nabla_{f} \cdot \sum_{k=1}^{18} \overline{\mathbf{u}_{d, k}^{\prime} q_{d, k}^{\prime}} \overline{\Delta p_{k}}
$$
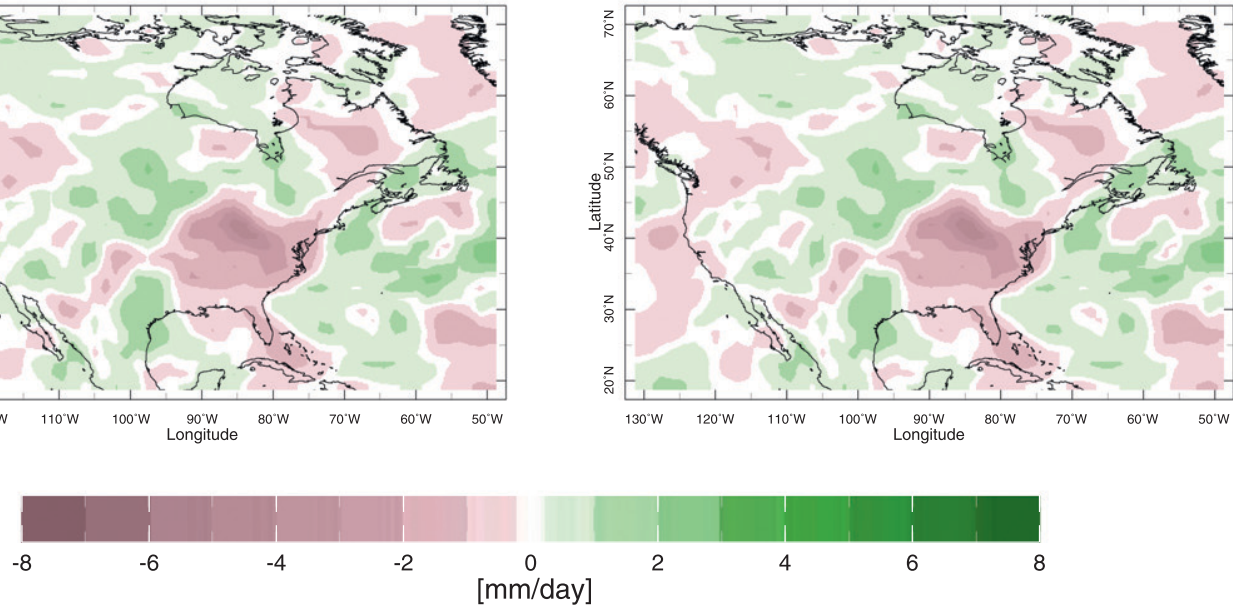

FIG. 10. Components of the MJJ 1993 moisture budget anomaly. The contribution from anomalies in the mean flow and mean humidity evaluated with (top left) 26 and (top right) 18 levels and the contribution from transient eddy moisture flux convergence evaluated with (middle) 6-hourly data and (bottom) daily data for (left) 26 and (right) 18 levels. (Units are $\mathrm{mm} \mathrm{day}^{-1}$.) 

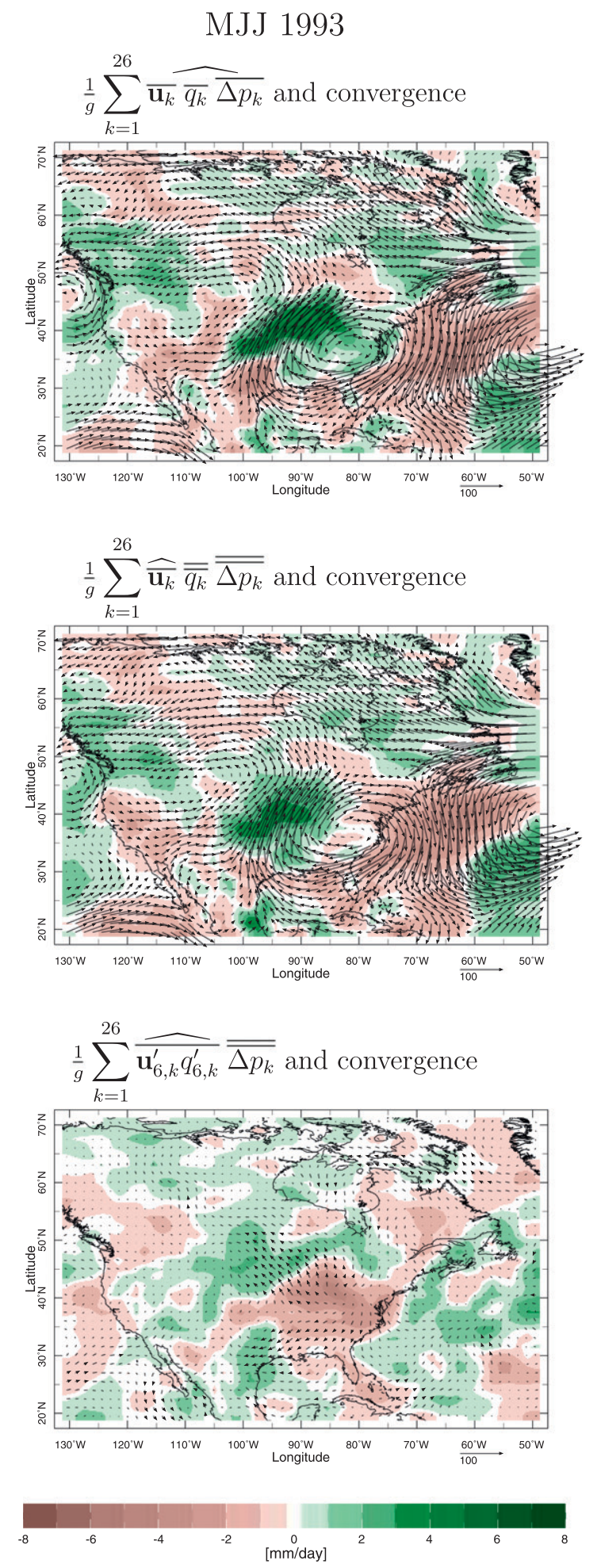

FIG. 11. (top) The MJJ 1993 mean flow moisture flux anomaly and its convergence evaluated with 26 levels, (middle) the part of this due to just mean flow anomalies combining with climatological humidity together with its convergence, and (bottom) the transient eddy moisture fluxes and their convergence evaluated with climatological pressure thicknesses. (Units are $\mathrm{kg}^{-1} \mathrm{~m}^{-1} \mathrm{~s}^{-1}$ for the fluxes and $\mathrm{mm} \mathrm{day}^{-1}$ for the convergence.) combining with the climatological humidity field and its associated vectors. The similarity of these two sets of fluxes and convergences indicates clearly that the circulation anomaly is the prime contributor to the $P-E$ anomaly while changes in humidity are less important (but not trivial). This result emphasizes the atmospheric dynamical origin of the MJJ 1993 flood in agreement with earlier studies (Mo et al. 1995; Liu et al. 1998). Figure 11 also shows the vectors of the transient eddy moisture flux together with their convergence (repeated from Fig. 10), which reveal the northwestward flux of moisture by the eddies from the southeast United States toward the upper Mississippi valley.

It is also of interest how the mean flow moisture convergence anomaly is contributed to by the divergent flow (and balancing vertical motion) and by moisture advection as in Eqs. (15) and (27). In this case we rewrite Eq. (33), with the help of Eqs. (14) and (15), and replace the pressure thicknesses with climatological values as

$$
\begin{aligned}
& \widehat{(\overline{P-E})_{\mathrm{der}}} \approx-\frac{1}{g \rho_{w}} \sum_{k=1}^{K} \overline{\left(\bar{q}_{k} \nabla_{f} \cdot \overline{\mathbf{u}}_{k}\right.}+\overline{\left.\overline{\mathbf{u}}_{k} \cdot \nabla_{f} \bar{q}_{k}\right)} \overline{\overline{\Delta p_{k}}} \\
& -\frac{1}{g \rho_{w}} \nabla_{f} \cdot \sum_{k=1}^{K} \overline{\left(\overline{\mathbf{u}_{6, k}^{\prime} q_{6, k}^{\prime}}\right)} \overline{\overline{\Delta p_{k}}}-\widehat{\mathrm{SFC}}_{K} .
\end{aligned}
$$

To perform this breakdown, the divergence operator has to be brought inside the vertical integration, and hence the surface term $\mathrm{SFC}_{K}$ is reintroduced. Figure 12 shows this breakdown for MJJ 1993. In the left column, we once more show the total anomalous convergence (mean plus transient flows) of vertically integrated moisture at the top (repeated from Fig. 10), and below it is the anomalous vertical integral of the total moisture convergence and the surface term $\mathrm{SFC}_{K}$. As for the climatological case (Fig. 7), the pattern and amplitude of anomalous moisture convergence is preserved whether the convergence is performed before or after the vertical integral. However, as before, the surface term is nonnegligible over the North American continent because of the presence of sizable surface pressure gradients. In the right column of Fig. 12, we show the total anomalous mean flow moisture convergence once more and its breakdown into a part due to the divergent mean flow and a part due to mean flow advection across mean humidity gradients. Both terms are important with clear roles for the term involving the mean flow convergence and ascending air in the region of highest $P-E$ anomaly in the Mississippi valley and for the moisture advection term farther to the east. The advection term here includes the advection of the mean specific humidity field by the anomalous flow and, referring to Fig. 11, the 
MJJ 1993
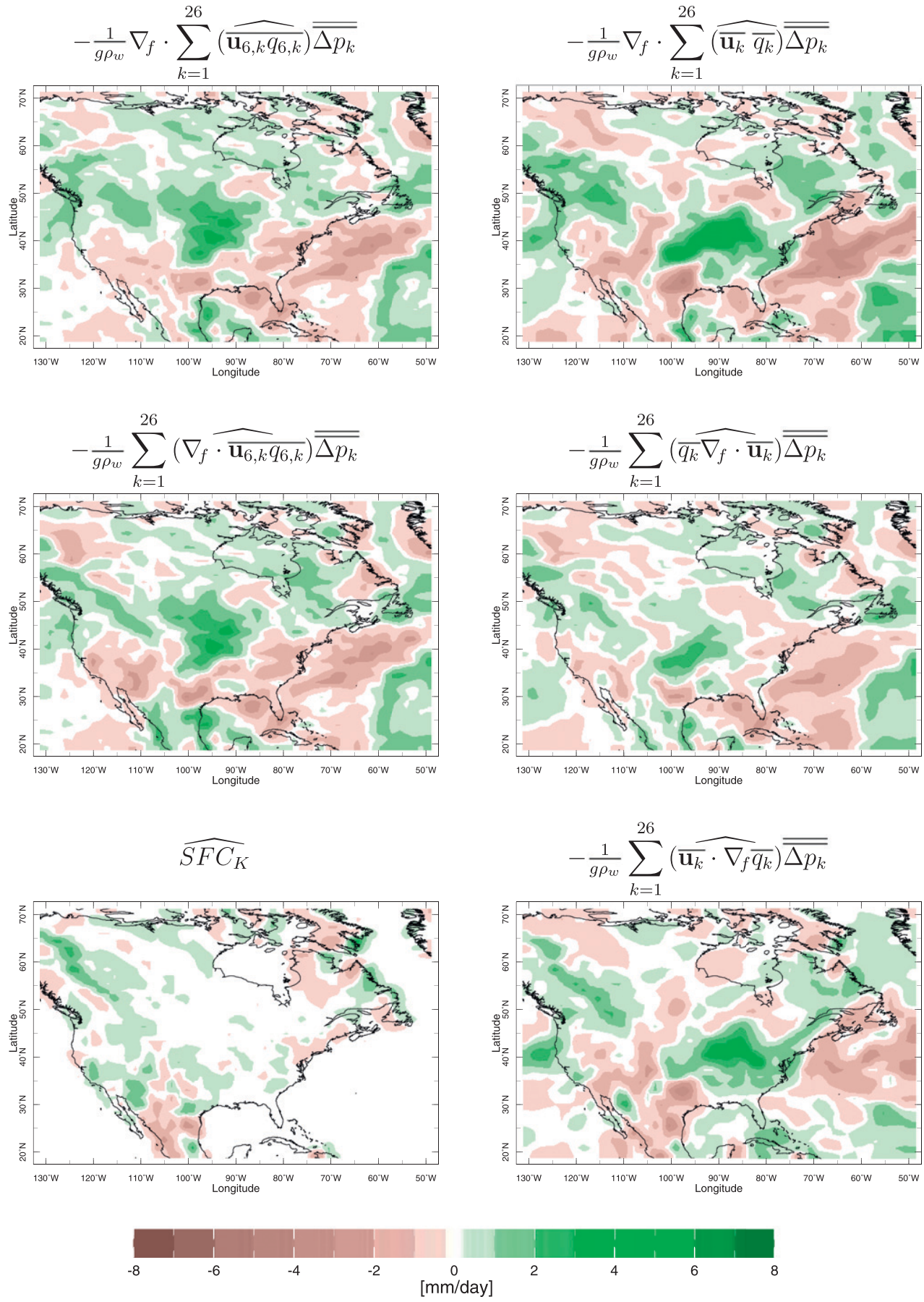

FIG. 12. (top left) The MJJ 1993 anomaly of the total convergence of the vertically integrated moisture flux and its breakdown into the vertical integral of (middle left) moisture convergence and (bottom left) the surface term all using 6-hourly data and 26 levels. (right) Terms related to the mean flow and mean humidity anomalies. (top right) The anomaly of the convergence of vertically integrated mean flow moisture flux and the components of the vertically integrated moisture convergence due to (middle right) the mean flow convergence and (bottom right) mean flow advection of mean humidity. All terms were evaluated using climatological pressure thicknesses. (Units are $\mathrm{kg}^{-1} \mathrm{~m}^{-1} \mathrm{~s}^{-1}$ for the fluxes and $\mathrm{mm} \mathrm{day}{ }^{-1}$ for the convergence.) 
strong southerly component to the flow anomalies in MJJ 1993 would create a positive $P-E$ tendency in that way.

Finally for the analysis of the MJJ 1993 Mississippi valley floods, we examine how well the anomalies would be captured if only 8 levels of daily data were available, as is common for CMIP archives of daily data. The 8-layer version quite reasonably captures the 26-level version of the total moisture convergence (Fig. 13). The errors introduced are quite random spatially but, in general, are of the magnitude of the field itself.

In summary, with 6-hourly data and care and attention in the performance of divergence operators and vertical integrals, as well as their order of computation, the diagnosed moisture budget can be analyzed and broken down to yield important insights into the causes of major hydroclimate anomalies such as the MJJ 1993 Mississippi floods. Nonetheless, in this case of the MJJ 1993 floods, even an analysis of causes based on just 8 levels of daily data might lead to useful, if not definitive, results.

\section{Conclusions}

The ability to diagnose moisture budget variations, and their causes, within reanalyses and atmosphere models, using archived data has been evaluated. The work was performed using the ERA-Interim data, which report vertically integrated moisture fluxes and convergences. This allows an assessment of errors introduced by diagnostically evaluating these terms from the archived data. The climatological moisture budget is evaluated as well as anomalies during the Mississippi valley flood of May-July 1993. Because of the assimilation procedure, ERA-Interim does not have a closed moisture budget and precipitation minus evaporation $P-E$ does not balance the vertically integrated moisture convergence and tendency. However, in diagnostic use of data from climate models, where this balance is more closely assured because of lack of data assimilation, the problem is always the evaluation of the vertically integrated moisture convergence. Hence here we focus on the evaluation of that using the ERA-Interim as our test case. Conclusions are as follows:

- Estimating the ERA-Interim-reported vertically integrated moisture convergence by applying a centered finite difference scheme to the ERA-Interim-reported vertically integrated moisture fluxes introduces significant error, which is greater over land than ocean. Errors are smaller when data closer to the ECMWF model resolution are used but do not disappear. The errors are probably partly due to the use of different numerical methods to evaluate the ERA-Interim-reported convergence of vertically integrated moisture fluxes to those used in our diagnostic evaluation of moisture convergence. However, since the ECMWF model itself uses yet different methods to update its moisture field and since the effects of moisture diffusion in the ERA-Interim cannot be diagnosed, some level of imbalance between diagnosed moisture convergence, $P-E$, and change in moisture storage has to be accepted.

- In midlatitudes where transient eddies cause significant time-averaged covariances of flow and humidity and hence time-averaged moisture fluxes and convergence, use of 6-hourly data introduces far less error than daily data. The error from using daily data appears as an underestimation of transient eddy moisture fluxes and convergence.

- Using 18 vertical levels instead of 26 vertical levels, with loss of vertical resolution in the boundary layer, introduces additional errors primarily over land areas and has little effect over the ocean, presumably because of differences in the complexity of the vertical structure of winds and humidity. However, going from 18 levels to the 8 levels common in CMIP archives of daily data introduces additional errors, which are now spread across both land and ocean. Monthly mean data in CMIP archives are usually stored at greater vertical resolution. Calculating the mean flow moisture convergence at the higher resolution and the transient contribution at the reduced vertical resolution will reduce error.

- Daily surface pressure data is not always available in model archives. However, performing vertical integrals with monthly mean pressure fields does not cause a significant increase in error compared to performing vertical integrals each day with daily pressure fields or every $6 \mathrm{~h}$ with 6-hourly pressure fields.

- When breaking down mean flow moisture convergence into components due to mass flux convergence and advection, the divergence operator has to be taken inside the vertical pressure integral, which introduces a surface term $q_{s} \mathbf{u}_{s} \cdot \nabla p_{s}$. A method is developed to numerically evaluate the vertical integral of mean flow moisture convergence and the surface term that assures that these sum exactly to equal the convergence of the vertically integrated moisture flux.

- Errors in diagnostically evaluating moisture budgets for particular seasons are no larger-and maybe smaller-than for climatological moisture budgets. This ensures that diagnosed moisture budgets can be reasonably examined to determine the causes of hydroclimate anomalies.

- The anomalous moisture budget evaluation was illustrated for the case of the Mississippi floods of 


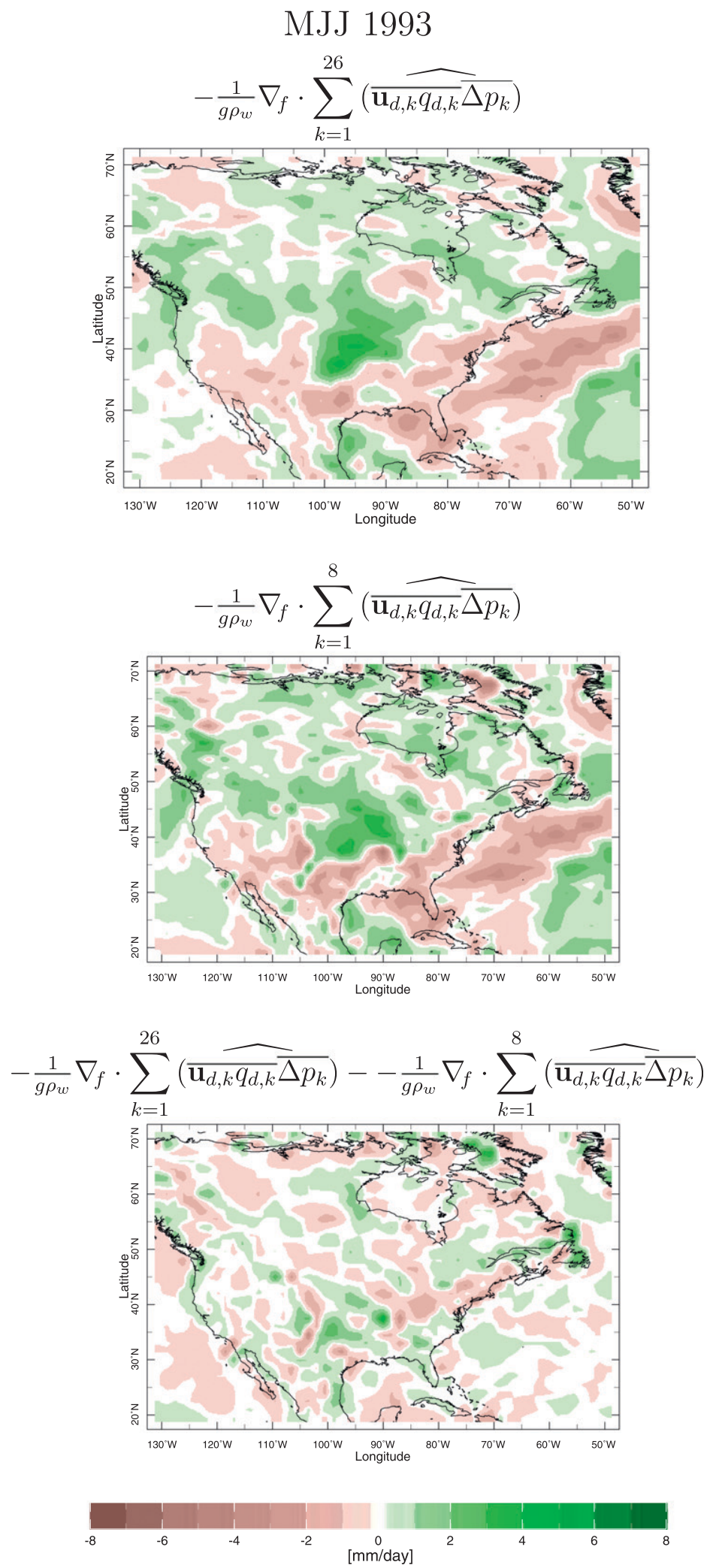

FIG. 13. The MJJ 1993 anomaly of the convergence of the total vertically integrated moisture flux computed with (top) 26 and (middle) 8 layers and (bottom) their difference. (Units are mm day ${ }^{-1}$.) 
May-July 1993. The diagnostically computed moisture convergence closely matches the ERA-Interimreported one as well as the ERA-Interim $P-E$. It is shown that mean flow moisture convergence related to a southerly flow anomaly and convergent flow was responsible for the positive $P-E$ in the central United States, while an anomalous transient eddy moisture flux divergence dried the southeast United States and transient eddy moisture flux convergence moistened the upper Mississippi valley. It is also shown that the moisture budget anomalies responsible for the flood were largely caused by circulation anomalies combining with the mean flow with the impacts of humidity anomalies being weaker. The contribution of the circulation anomalies was effected through both changes in mass convergence (and hence vertical motion) and changes in the advection of the mean humidity. The transient eddy contribution to the anomaly was underestimated with hourly data. However, an analysis with even 8 levels of daily data would reveal the major causes of the flood.

\section{Recommendation}

In this regard, we make the following recommendation: Climate models and reanalyses should compute covariances at the model time step and then average these into monthly means (e.g., archive monthly means of $u_{T, k} q_{T, k}$, where $T$ refers to time-step values on the model vertical grid) for archiving in, for example, CMIP data and in reanalysis data.

Monthly mean flow-humidity covariances can be vertically integrated with the monthly pressure fields to yield an accurate approximation to the total monthly mean convergence of vertically integrated moisture fluxes. With this saved, the transient contributions can be evaluated by subtracting the monthly mean contributions evaluated from the monthly mean data. Transient contributions estimated in this way will in fact be more accurate than those computed with archived 6-hourly data and even more accurate than those computed with daily data at the modest cost of increasing the size of model data archives. If this was done, it would help researchers perform accurate analyses of the atmospheric branch of the hydrological cycle and further advance knowledge and prediction of the earth's climate system.

Acknowledgments. This work was supported by NOAA Award NA10OAR4310137 (Global Decadal Hydroclimate Variability and Change). We thank Yochanan Kushnir and Paul Berrisford (ECMWF) for useful discussions, Donna Lee for downloading the ERA-Interim data, and the European Centre for Medium-Range Weather Forecasts for making the reanalysis data available.

\section{REFERENCES}

Allen, M. R., and W. J. Ingram, 2002: Constraints on future changes in climate and the hydrologic cycle. Nature, 419, 224-232.

Berrisford, P., P. Kallberg, S. Kobayashi, D. Dee, S. Uppala, A. J. Simmons, P. Poli, and H. Sato, 2011a: Atmospheric conservation properties in ERA-Interim. Quart. J. Roy. Meteor. Soc., 137, 1381-1399.

, and Coauthors, 2011b: The ERA-Interim archive version 2.0. European Centre for Medium-Range Weather Forecasts ERA Rep. 1, 23 pp.

Cutter, S. L., C. T. Emrich, J. J. Webb, and D. Morath, 2009: Social vulnerability to climate variability hazards: A review of the literature. University of South Carolina Hazards and Vulnerability Research Institute Tech. Rep., 44 pp.

Dee, D., and Coauthors, 2011: The ERA-Interim reanalysis: Configuration and performance of the data assimilation system. Quart. J. Roy. Meteor. Soc., 137, 553-597.

ECMWF, 2012: IFS documentation Cy37r2 operational implementation 18 May 2011. Part III: Dynamics and numerical procedures. European Centre for Medium-Range Weather Forecasts Tech. Rep., 29 pp.

Harnik, N., R. Seager, N. Naik, M. Cane, and M. Ting, 2010: The role of linear wave refraction in the transient eddy-mean flow response to tropical Pacific SST anomalies. Quart. J. Roy. Meteor. Soc., 136, 2132-2146.

Held, I. M., and B. J. Soden, 2006: Robust responses of the hydrological cycle to global warming. J. Climate, 19, 5686-5699.

Huang, H., R. Seager, and Y. Kushnir, 2005: The 1976/77 transition in precipitation over the Americas and the influence of tropical SST. Climate Dyn., 24, 721-740.

Konor, C. S., and A. Arakawa, 1997: Design of an atmospheric model based on a generalized vertical coordinate. Mon. Wea. Rev., 125, 1649-1673.

Liu, A. Z., M. Ting, and H. Wang, 1998: Maintenance of circulation anomalies during the 1988 drought and 1993 floods over the United States. J. Atmos. Sci., 55, 2810-2832.

Mo, K. C., J. Nogues-Paegle, and J. Paegle, 1995: Physical mechanisms of the 1993 summer floods. J. Atmos. Sci., 52, 879-895.

Nakamura, J., U. Lall, Y. Kushnir, A. W. Robertson, and R. Seager, 2013: Dynamical structure of major spring floods in the U.S. Midwest and the United Kingdom. J. Hydrometeor., 14, 485-504.

Seager, R., and G. A. Vecchi, 2010: Greenhouse warming and the 21st century hydroclimate of southwestern North America. Proc. Natl. Acad. Sci. USA, 107, 21277-21 282.

— tecting recent anthropogenic hydroclimate change. J. Climate, 25, 236-261.

—, N. Harnik, W. A. Robinson, Y. Kushnir, M. Ting, H. P. Huang, and J. Velez, 2005: Mechanisms of ENSO-forcing of hemispherically symmetric precipitation variability. Quart. J. Roy. Meteor. Soc., 131, 1501-1527.

— , and Coauthors, 2007: Model projections of an imminent transition to a more arid climate in southwestern North America. Science, 316, 1181-1184.

, N. Naik, M. A. Cane, N. Harnik, M. Ting, and Y. Kushnir, 2010a: Adjustment of the atmospheric circulation to tropical Pacific SST anomalies: Variability of transient eddy 
propagation in the Pacific-North America sector. Quart. J. Roy. Meteor. Soc., 136, 277-296.

, and G. A. Vecchi, 2010b: Thermodynamic and dynamic mechanisms for large-scale changes in the hydrological cycle in response to global warming. J. Climate, 23, 4651-4668.

- _ - and L. Vogel, 2012: Does global warming cause intensified interannual hydroclimate variability? J. Climate, $\mathbf{2 5}$, 3355-3372.

Seneviratne, S. I., P. Viterbo, D. Luthi, and C. Schar, 2004: Inferring changes in terrestrial water storage using ERA-40 reanalysis data: The Mississippi River basin. J. Climate, 17, 2039-2057.

Solomon, S., D. Qin, M. Manning, M. Marquis, K. Averyt, M. M. B. Tignor, H. L. Miller Jr., and Z. Chen, Eds., 2007: Climate Change 2007: The Physical Science Basis. Cambridge University Press, 996 pp.

Taylor, K. E., R. J. Stouffer, and G. A. Meehl, 2012: An overview of CMIP5 and the experiment design. Bull. Amer. Meteor. Soc., 93, 485-498.
Trenberth, K., 1997: Using atmospheric budgets as a constraint on surface fluxes. J. Climate, 10, 2796-2809.

— , and C. J. Guillemot, 1995: Evaluation of the global atmospheric moisture budget as seen from analyses. J. Climate, $\mathbf{8}$, 2255-2272.

—, and —, 1996: Physical processes involved in the 1988 drought and 1993 floods in North America. J. Climate, 9, 12881298.

— hydrological cycle in the NCEP/NCAR reanalyses. Climate Dyn., 14, 213-231.

— J. T. Fasullo, and J. Mackaro, 2011: Atmospheric moisture transport from ocean to land and global energy flows in reanalyses. J. Climate, 24, 4907-4924.

Weaver, S. J., A. Ruiz-Barradas, and S. Nigam, 2009: Pentad evolution of the 1988 drought and 1993 flood over the Great Plains: An NARR perspective on the atmospheric and terrestrial water balance. J. Climate, 22, 5366-5384. 